Article

\title{
Synthesis of Polyaniline-Coated Graphene

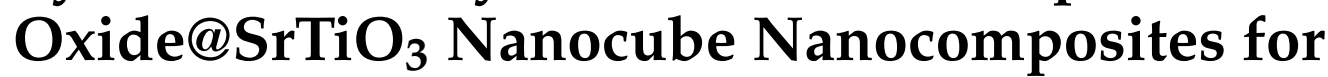 Enhanced Removal of Carcinogenic Dyes from Aqueous Solution
}

\author{
Syed Shahabuddin ${ }^{1}$, Norazilawati Muhamad Sarih ${ }^{1, *}$, Muhammad Afzal Kamboh ${ }^{1}$, \\ Hamid Rashidi Nodeh ${ }^{1,2}$ and Sharifah Mohamad ${ }^{1,3}$ \\ 1 Polymer Research Laboratory, Chemistry Department, Faculty of Science, University of Malaya, \\ Kuala Lumpur 50603, Malaysia; syedshahabuddin@siswa.um.edu.my (S.S.); \\ afzal82_kamboh@yahoo.com (M.A.K.); hamid_rashidi82@yahoo.com (H.R.N.); \\ sharifahm@um.edu.my (S.M.) \\ 2 Department of Chemistry, Faculty of Science, University of Tehran, Tehran 14174, Iran \\ 3 University of Malaya Centre for Ionic Liquids (UMCiL), University of Malaya, \\ Kuala Lumpur 50603, Malaysia \\ * Correspondence: nmsarih@um.edu.my; Tel.: +60-3-7967-7173
}

Academic Editor: Changle Chen

Received: 11 June 2016; Accepted: 4 August 2016; Published: 2 September 2016

\begin{abstract}
The present investigation highlights the synthesis of polyaniline (PANI)-coated graphene oxide doped with $\mathrm{SrTiO}_{3}$ nanocube nanocomposites through facile in situ oxidative polymerization method for the efficient removal of carcinogenic dyes, namely, the cationic dye methylene blue (MB) and the anionic dye methyl orange (MO). The presence of oxygenated functional groups comprised of hydroxyl and epoxy groups in graphene oxide (GO) and nitrogen-containing functionalities such as imine groups and amine groups in polyaniline work synergistically to impart cationic and anionic nature to the synthesised nanocomposite, whereas $\mathrm{SrTiO}_{3}$ nanocubes act as spacers aiding in segregation of GO sheets, thereby increasing the effective surface area of nanocomposite. The synthesised nanocomposites were characterised by field emission scanning electron microscopy (FESEM), transmission electron microscopy (TEM), thermogravimetric analysis (TGA), X-ray diffraction (XRD), and Fourier transform infrared spectroscopy (FTIR). The adsorption efficiencies of graphene oxide (GO), PANI homopolymer, and $\mathrm{SrTiO}_{3}$ nanocubes-doped nanocomposites were assessed by monitoring the adsorption of methylene blue and methyl orange dyes from aqueous solution. The adsorption efficiency of nanocomposites doped with $\mathrm{SrTiO}_{3}$ nanocubes were found to be of higher magnitude as compared with undoped nanocomposite. Moreover, the nanocomposite with $2 \mathrm{wt} \% \mathrm{SrTiO}_{3}$ with respect to graphene oxide demonstrated excellent adsorption behaviour with $99 \%$ and $91 \%$ removal of $\mathrm{MB}$ and $\mathrm{MO}$, respectively, in a very short duration of time.
\end{abstract}

Keywords: graphene oxide; polyaniline; nanocomposites; adsorbent; methylene blue; methyl orange

\section{Introduction}

Water pollution poses a serious threat to the environment, thereby attracting much scientific attention to the removal of organic waste and toxic water pollutants from aqueous bodies [1]. The textile industry, one of the major worldwide contributors to water pollution, causes major impact on the quality of available water resources through deliberate or inadvertent release of dye effluents into water bodies. Dyes are complex organic molecules that adhere to the surface of fabrics, thereby imparting colour to them. There are more than 100,000 commercially available dyes [1] used in a 
wide variety of application including textiles [2], paper [3], tanning industries [4], plastics, printing, food processing [5-7], and so on. Approximately 10,000 tonnes of synthetic dyes are used per year by textile industries alone, discharging nearly 100 tonnes of dyes in water bodies as effluents [7]. Most of the synthetic dyes evade conventional water treatment methods, thereby accumulating in the environment due to their high degree of stability towards biodegradation, temperature, light, detergents, and soaps [8,9]. Methylene blue (MB) and methyl orange (MO), commercial dyes used for various applications such as textiles, papers, leathers, additives, laser printing, etc., are heterocyclic aromatic chemical compounds having complex chemical structures and synthetic origin, owing to which they are resistant to biodegradation and very stable to light and oxidation [10-12]. These dyes are highly toxic, persistent, carcinogenic, and mutagenic in nature. By virtue of their cationic/anionic as well as aromatic nature they are easily soluble in an aqueous/alcoholic medium and usually generate sulphur/nitric oxides at high temperature. As a result of the reduction process, these dyes reduce the dissolved oxygen, which modifies the properties as well as characteristics of aqueous fluids and can cause severe adverse health effects such as breathing difficulties, nausea, vomiting allergic dermatitis, skin irritation, cancer, and mutations [1]. Hence, for a safer environment, the removal of these noxious dyes from aqueous environment is essential. Up to now considerable efforts such as coagulation [13], photocatalysis [10,12], biological treatment [14], chemical oxidation [15], membrane separation [16], and adsorption $[17,18]$ have been performed to eliminate noxious dyes from aqueous environment. Among all these techniques, adsorption continues to attract considerable attention due to its simplistic approach and numerous benefits such as greater efficiency, the capacity to remove dyes on a large scale, the ease of recovery, and the recyclability of adsorbents. Different classes of adsorbents such as activated carbon [19], polymeric materials [20], biomass [21], MWCNT [22], etc. have been employed to eliminate dyes from polluted water.

Presently, conducting polymers have been the focus of immense scientific attention at an academic and industrial level. Unique electrical and optoelectronic properties due to extended $\pi$-conjugated electron systems make conductive polymers extensively explored materials. Conducting polymers such as polythiophene, polyacetylene, polypyrrole, polyphenylene, and polyaniline have been widely studied in multidisciplinary research areas comprising environmental, electronics, electromagnetic, thermoelectric, sensors, batteries, electro-luminescence, and electromechanical applications [23-28]. Among the conducting polymer family, polyaniline (PANI), owing to its unique electrochemical properties, higher environmental stability, easy synthetic methodologies, cost-effectiveness, efficient thermal stability, and wide varieties of application, has been most intensively investigated by the scientific community [29]. However, several drawbacks such as poor solubility, poor mechanical properties, lower effective surface area, etc. restrict the use of PANI in many environmental applications [30]. In order to overcome these limitations, PANI is often polymerised in the presence of variety of other organic and inorganic materials to enhance its properties. Morphology and active surface area are two major characteristics that play a significant role in increasing the adsorption capacity of PANI-based composite materials; they can be manipulated by incorporating nanoscale materials in the matrix of the polymer. PANI-based nanocomposites have been extensively studied as adsorbent materials for the removal of dyes and other organic pollutants from waste waters and continue to be the most favoured contender for various environmental applications [31].

Graphene, a two-dimensional one-atom-thick sheet of all sp²-hybridized carbon, has received research interest due to its distinctive electronic, thermal, optical, mechanical, and excellent chemical tolerance capabilities, as well as its large surface-to-volume ratio [32-35]. One of the most attractive features of graphene is its large theoretical specific surface area $\left(2630 \mathrm{~m}^{2} \cdot \mathrm{g}^{-1}\right)$, which makes it a suitable candidate for use as an adsorbent material [36]. Due to these distinctive properties, graphene is often used as an appropriate matrix for designing nanocomposites with other substances such as polymers [37], a metal-organic framework [38,39], metal nanoparticles [40], and so on. PANI nanocomposites with graphene oxide (GO) have demonstrated enhanced physical and chemical properties compared with neat PANI or graphene oxide and have been exploited in numerous 
applications [40-43]. Besides GO, inorganic metal oxide-based nanocomposites play a significant role in potential applications including photodegradation, waste water treatment through adsorption, photovoltaics, photochromism, etc. owing to their low cost, facile synthesis, large surface area, and physiochemical properties [44]. Various nanocomposite materials with inorganic metal oxides such as $\mathrm{SrTiO}_{3}$ [12], $\mathrm{TiO}_{2}$ [45], $\mathrm{Fe}_{3} \mathrm{O}_{4}$ [46], $\mathrm{Co}_{3} \mathrm{O}_{4}$ [10,47], etc. have been employed for the effective treatment of dye waste water. Therefore, GO and PANI, together with some metal oxide nanoparticles, can be explored for the synthesis of nanocomposite materials to address the present-day issue of water pollution.

In the present study we reported the facile synthesis of polyaniline-coated graphene oxide doped with $\mathrm{SrTiO}_{3}$ nancocube nanocomposites, synthesised through a simple in situ oxidative polymerisation technique for the adsorption of a cationic dye (MB) and an anionic dye (MO). GO was synthesised using modified Hummer's method, whereas $\mathrm{SrTiO}_{3}$ nanocubes were synthesized using the simplistic hydrothermal technique and both were later incorporated into the polymer matrix during polymerization. Simultaneous removal of both cationic and anionic dyes by adsorbent is not easily achieved as the adsorbent must have the ability to attract both negative and positively charged particles. Here graphene oxide, due to the presence of oxygen-containing functionalities, attains a negative charge whereas polyaniline, due to the presence of nitrogen-containing functionalities (imine group $-\mathrm{N}=$ and amine group $-\mathrm{N}<$ ), develops a positively-charged backbone, thereby causing intensive electrostatic attraction between the nanocomposites and dye molecules. $\mathrm{SrTiO}_{3}$ nanocubes act as spacers, which help in the segregation of GO sheets, thereby increasing the overall effective surface area of the nanocomposites. Therefore, $\mathrm{GO}$ and $\mathrm{PANI}$, along with $\mathrm{SrTiO}_{3}$ nanocubes, act synergistically, imparting desired properties such as zwitterionic nature and enhanced surface area to the nanocomposites. Easy preparation, cheaper costs and superior adsorption capacity for anionic and cationic dyes projects these nanocomposites as the potential adsorbent material for waste water treatment.

\section{Experimental Section}

\subsection{Materials}

Aniline (Fluka, St. Louis, MO, USA, $\geq 99 \%$ ) was distilled under reduced pressure and stored in the dark before use. Ammonium peroxydisulfate (APS) (Merck, Kenilworth, NJ, USA, $\geq 99 \%$ ), strontium hydroxide octahydrate (Sigma Aldrich, St. Louis, MO, USA, 95\%); titanium(IV) oxide, anatase (Sigma Aldrich, St. Louis, MO, USA, 99.7\%), hydrochloric acid, $\mathrm{HCl}$ (Merck, Kenilworth, NJ, USA, 37\%), sulphuric acid, $\mathrm{H}_{2} \mathrm{SO}_{4}$ (Sigma Aldrich, St. Louis, MO, USA, 98\%), methanol (Merck, Kenilworth, NJ, USA, 99.9\%), potassium permanganate (Sigma Aldrich, St. Louis, MO, USA, $\geq 99 \%$ ), hydrogen peroxide (Sigma Aldrich, St. Louis, MO, USA, 30\%), graphite powder (Sigma Aldrich, St. Louis, MO, USA, $\geq 99.99 \%$ ), and ammonia solution (R \& M Chemicals, Essex, UK, 25\%) were used as received without further purification. All of the reagents that were involved in the experiments were of analytical grade. Deionised water (DI) was used throughout the entire study.

\subsection{Synthesis of Graphene Oxide (GO)}

GO was synthesised using a modified Hummer's method, as reported elsewhere [48]. Briefly, concentrated sulphuric acid $(120 \mathrm{~mL})$ was added into a three-neck round bottom flask $(500 \mathrm{~mL})$ immersed in ice bath under continuous stirring at $500 \mathrm{rpm}$. Five grams of graphite powder were added into ice-cooled concentrated sulphuric acid, followed by subsequent addition of $2.5 \mathrm{~g} \mathrm{NaNO}_{3}$ and $15 \mathrm{~g}$ $\mathrm{KMnO}_{4}$. After a while the ice bath was removed and the reaction mixture was stirred overnight. When the colour of the reaction mixture turned light grey and converted into a paste, $150 \mathrm{~mL}$ of deionised water were added gradually into the mixture to dilute the paste. At this point the temperature of the reaction vessel was raised to $98{ }^{\circ} \mathrm{C}$ under continuous stirring for $2 \mathrm{~h}$. Then $50 \mathrm{~mL}$ of $\mathrm{H}_{2} \mathrm{O}_{2}$ were added and the reaction mixture was stirred for $30 \mathrm{~min}$. The reaction mixture was then filtered and washed 
with $5 \% \mathrm{HCl}$ until the filtrate became colourless. Later it was washed with ethanol and subsequently with deionized water until the filtrate became neutral, and dried in a vacuum oven at $60^{\circ} \mathrm{C}$.

\subsection{Synthesis of $\mathrm{SrTiO}_{3}$ Nanocubes}

A typical hydrothermal technique was utilised for the synthesis of $\mathrm{SrTiO}_{3}$ nanocubes, as reported previously [12]. In summary, a calculated amount of strontium hydroxide octahydrate (1.4 g) was dissolved in $20 \mathrm{~mL} \mathrm{NaOH}(3 \mathrm{M})$ under constant stirring. To this solution, titanium dioxide solution prepared by mixing $0.4 \mathrm{~g}$ of $\mathrm{TiO}_{2}$ in $20 \mathrm{~mL} \mathrm{NaOH}(3 \mathrm{M})$ was added dropwise at a rate of one drop per second with vigorous stirring. After $30 \mathrm{~min}$ of stirring, $40 \mathrm{~mL}$ of the reaction mixture was transferred to a $100-\mathrm{mL}$ Teflon-lined stainless steel autoclave and subjected to hydrothermal treatment at $130{ }^{\circ} \mathrm{C}$ for $72 \mathrm{~h}$. The obtained precipitate of $\mathrm{SrTiO}_{3}$ nanocubes was then washed thoroughly with DI water several times and dried in a vacuum oven at $60^{\circ} \mathrm{C}$ for $24 \mathrm{~h}$.

\subsection{Synthesis of Polyaniline (PANI)}

Polyaniline was synthesised by the oxidative polymerisation of distilled aniline, which was dissolved in aqueous $\mathrm{HCl}(1 \mathrm{M})$, using ammonium persulfate (APS) as an oxidant. Aniline (0.0215 mol) was dissolved in $30 \mathrm{~mL}$ of an aqueous solution of $\mathrm{HCl}(1 \mathrm{M})$, and APS $(0.0268 \mathrm{~mol})$ was dissolved in $35 \mathrm{~mL} \mathrm{HCl}(1 \mathrm{M})$. The oxidant solution was then added slowly to the aniline solution with continuous stirring at $25{ }^{\circ} \mathrm{C}$. The reaction mixture was stirred continuously for two hours. The reaction mixture was then filtered and washed with $\mathrm{HCl}(0.5 \mathrm{M})$ until the filtrate became colourless and subsequently with DI water until the filtrate became neutral. The obtained polymer was dried in a vacuum oven at $60{ }^{\circ} \mathrm{C}$ overnight. The green colour of the obtained polymer indicated the formation of conductive polyaniline emeraldine salt.

\subsection{Synthesis of PANI-Coated GO (GOPSr-0)}

PANI-coated GO nanocomposite was prepared by in situ oxidative polymerization of aniline by keeping the feed ratio of aniline to GO as 20:80. A calculated amount of aniline was dissolved in an aqueous solution of $\mathrm{HCl}(1 \mathrm{M})$. Then, a calculated amount of $\mathrm{GO}$ was dispersed in this solution and sonicated for $1 \mathrm{~h}$. Later on, the oxidant solution (mole ratio with aniline 1:1.25) in $1 \mathrm{M} \mathrm{HCl}$ was gradually added to the reaction mixture under vigorous stirring at room temperature. The reaction mixture was stirred continuously for two hours. The reaction mixture was then filtered and washed with $\mathrm{HCl}(0.5 \mathrm{M})$ until the filtrate became colourless and subsequently with DI water until the filtrate became neutral. The obtained composite was dried in a vacuum oven at $60{ }^{\circ} \mathrm{C}$ overnight and labelled as GOPSr-0. Scheme 1 represents the reaction pathway for the synthesis of GOPSr-0.

\subsection{Synthesis of $\mathrm{SrTiO}_{3}$ Nanocubes-Doped, PANI-Coated GO (GOPSr)}

$\mathrm{SrTiO}_{3}$ nanocube-doped, PANI-coated GO nanocomposites were prepared with different wt $\%$ of $\mathrm{SrTiO}_{3}\left(1,2\right.$, and $5 \mathrm{wt} \%$ with respect to GO). A calculated amount of $\mathrm{SrTiO}_{3}$ nanocubes was dispersed in $5 \mathrm{~mL}$ of deionised water by sonication and added dropwise to an aniline-GO suspension in $\mathrm{HCl}$ (as described in previous section) under vigorous stirring. The resulting mixture was sonicated for $1 \mathrm{~h}$ until it became uniform. The work-up procedure was the same as described in the previous section. The obtained nanocomposites were labelled as GOPSr-1, GOPSr-2, and GOPSr-5 indicating 1,2 , and $5 \mathrm{wt} \%$ of SrTiO3 nanocubes with respect to GO, respectively. Scheme 1 represents the graphical illustration of the reaction pathway for the synthesis of $\mathrm{SrTiO}_{3}$ nanocube-doped, PANI-coated GO nanocomposites.

\subsection{Characterisation Techniques}

The surface morphological and elemental analysis of the synthesised product was conducted using a JSM-7600F field emission scanning electron microscope (JEOL Ltd., Tokyo, Japan) operated at $10 \mathrm{kV}$. 
The size and shape of the obtained $\mathrm{SrTiO}_{3}$ nanocubes were studied using a JEM-2100F high-resolution transmission electron microscope (JEOL Ltd., Tokyo, Japan). X-ray diffraction (XRD) patterns were recorded using an Empyrean X-ray diffractometer (PAN analytical, Almelo, The Netherlands) from $2 \theta=10^{\circ}$ to $90^{\circ}$ using $\mathrm{Cu} \mathrm{K} \alpha$ radiations $(\lambda=1.5418 \AA)$ at a scan rate of $0.02 \mathrm{~s}^{-1}$. Fourier transform infrared (FT-IR) spectra of the powdered samples were recorded using a Perkin Elmer RX1 FT-IR ATR spectrometer (Perkin Elmer, Waltham, MA, USA) in the range of $400-4000 \mathrm{~cm}^{-1}$ in spectral-grade KBr pellets.

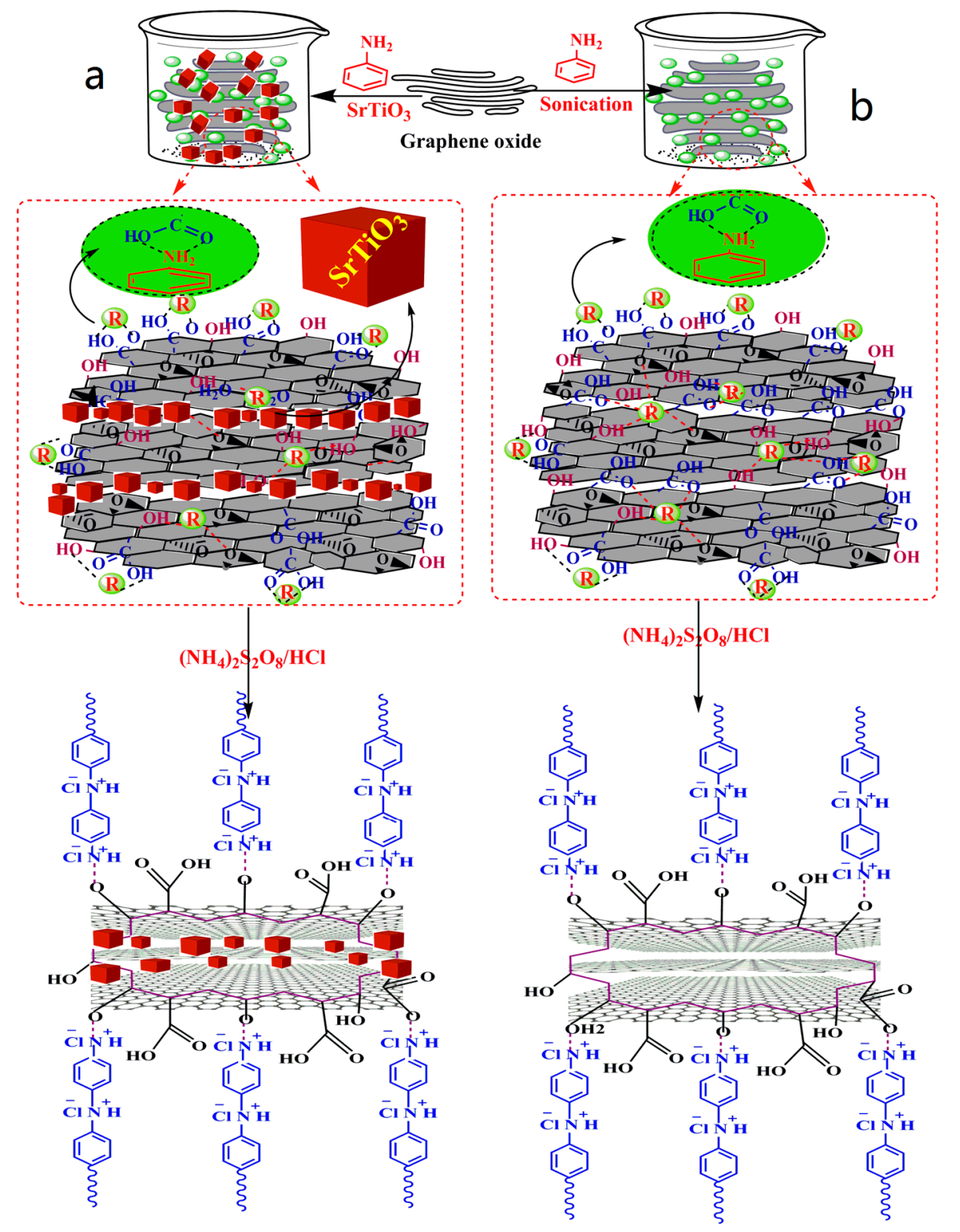

Scheme 1. Reaction pathway for the synthesis of PANI-coated GO nanocomposite (a) doped with $\mathrm{SrTiO}_{3}$ and (b) without $\mathrm{SrTiO}_{3}$ (dotted lines represent hydrogen bonding, $\pi-\pi$ interactions, and electrostatic interactions of PANI with GO).

\subsection{Dye Adsorption Study}

The adsorption studies were carried out using MB and MO as the model dyes in the water phase to investigate the adsorption ability of synthesised nanocomposites. The dye adsorption experiments were conducted at room temperature in a set of Erlenmeyer flasks by batch process to study the 
outcome of different parameters such as type of adsorbent $\left(\mathrm{SrTiO}_{3}\right.$ nanocubes, GO, PANI, GOPSr-0, GOPSr-1, GOPSr-2, and GOPSr-5), time (0-60 min), initial pH (4.5-9.5), and effect of $\mathrm{NaCl}$ concentration $(10-50 \mathrm{~g} / \mathrm{L})$. In general, $50 \mathrm{mg}$ of the dye was dissolved in $1 \mathrm{~L}$ of distilled water and the solution $\mathrm{pH}$ was adjusted by adding $\mathrm{HCl}(0.1 \mathrm{~N})$ or $\mathrm{NaOH}(0.1 \mathrm{~N})$. A calculated amount of adsorbent was added to $100 \mathrm{~mL}$ of dye solution and kept on a shaker with constant shaking at $180 \mathrm{rpm}$. Then, $3 \mathrm{~mL}$ of the dye suspension were withdrawn at a regular time interval and centrifuged. The UV-visible absorption spectra of the supernatant solution were analysed using a UV-visible spectrometer (Thermo Scientific Evolution, Thermo Fisher Scientific, Waltham, MA, USA) in 1-cm quartz cuvettes to monitor the characteristic absorption peak of $\mathrm{MB}$ and $\mathrm{MO}$. The percentage dye removal from the aqueous solution was determined according to the following equation:

$$
\% R=\left\{\left(C_{0}-C_{t}\right) / C_{0}\right\} \times 100,
$$

where $C_{0}$ is the initial concentration of the $\mathrm{MO}(\mathrm{mg} / \mathrm{L})$ and $C_{\mathrm{t}}$ is the concentration of $\mathrm{MO}(\mathrm{mg} / \mathrm{L})$ at time $t$.

\section{Results and Discussion}

\subsection{Synthesis}

The main goal of this study was to design a new highly segregated, PANI-coated GO nanocomposite doped with $\mathrm{SrTiO}_{3}$ nanocubes, bearing cationic as well as anionic functional sites for the enhanced removal of $\mathrm{MB}$ and $\mathrm{MO}$ dyes. To achieve the desired goal, an aniline monomer containing amine functionality was introduced on the surface of $\mathrm{GO}$, which was subsequently polymerized in the presence of ammonium persulphate in acidic medium in order to obtain a PANI-coated GO nanocomposite, as shown in Scheme 1. PANI-coated GO nanocomposites contain a positively charged polymeric backbone and $\mathrm{sp}^{2}$ hybridized framework along with anionic functionalities. However, due to stacking of GO sheets, PANI-coated GO nanocomposite possess a substantial amount of agglomeration, which reduces the effective surface area of the adsorbent. In order to enhanced the effective surface area of $\mathrm{SrTiO}_{3}$, nanocubes were incorporated into the matrix of PANI-coated GO nanocomposite, where they act as spacer molecules to segregate the GO sheets, thereby increasing the total effective surface area, a prime prerequisite for an efficient adsorbent (Scheme 2).

\subsection{Morphological Analysis of Nanocomposites}

The morphology and structure analysis of the synthesised graphene oxide, PANI, $\mathrm{SrTiO}_{3}$ nanocubes and GOPSr-2 nanocomposite were studied through FESEM. Figure 1a explicates the morphology of PANI, which displayed a specific flake-like structure. Figure $1 \mathrm{~b}$ reveals the surface morphology of GO, clearly illustrating the disorderly stacked, folded sheet-like accumulation. Figure 1c depicts the morphology of an $\mathrm{SrTiO}_{3}$ nanocube. As evident from the micrographs, $\mathrm{SrTiO}_{3}$ nanoparticles have attained cube-shaped morphology with approximately uniform particle size. Moreover, to confirm the formation of $\mathrm{SrTiO}_{3}$ nanocubes, the synthesised nanoparticles were analysed via TEM. Figure 1d represents the TEM image of $\mathrm{SrTiO}_{3}$ nanocubes, which visibly reveals the formation of cubic particles in nanoscale, thereby confirming the formation of $\mathrm{SrTiO}_{3}$ nanocubes. Figure 1e exhibits PANI-coated GO nanocomposite (GOPSr-0), which indicate the transformation in the surface morphology of GO upon coating by PANI. As is obvious from the figure, the folded and stacked GO sheets become segregated after being coated by PANI. This segregation was further enhanced by doping PANI-coated $\mathrm{GO}$ with $\mathrm{SrTiO}_{3}$, as illustrated by Figure 1f. The cubic shaped $\mathrm{SrTiO}_{3}$ nanoparticles can be seen on the surface of the nanocomposites embedded within the layers of GO and PANI, as demonstrated by Figures S1a and b. Figure S2 exhibits the TEM images of GOPSr-2 at different magnifications. As evident from the TEM micrographs, folded sheets of GO are coated by PANI, whereas $\mathrm{SrTiO}_{3}$ nanoparticles can be seen embedded within the GO layers. Therefore, coating of GO with PANI and doping with $\mathrm{SrTiO}_{3}$ nanocubes had completely transformed the surface 
morphology with much segregated composite material, thereby leading to the higher surface area, a prime prerequisite for an ideal adsorbent material.
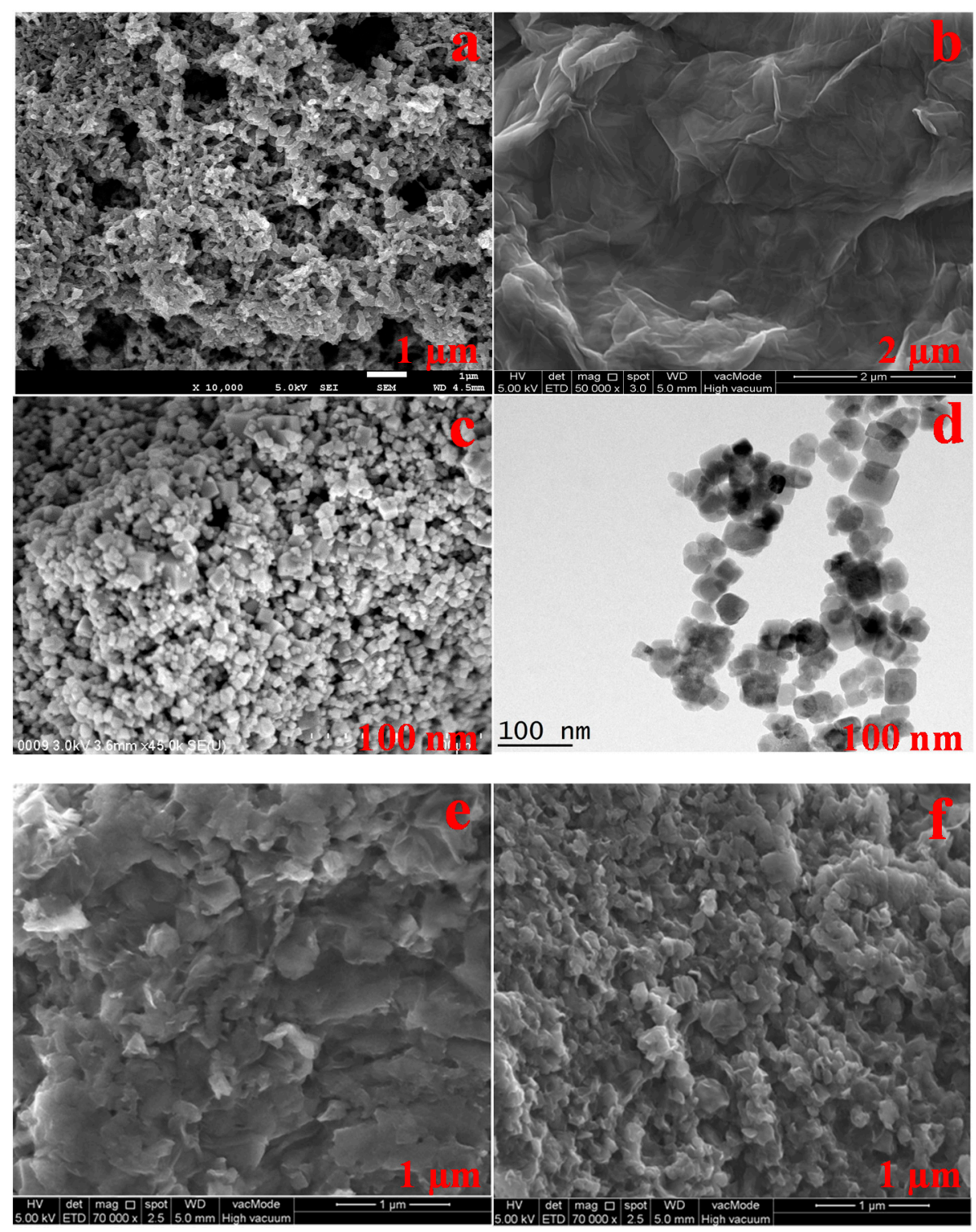

Figure 1. FESEM micrographs of (a) PANI; (b) GO; (c) $\mathrm{SrTiO}_{3}$ nanocubes; (e) GOPSr-0; and (f) GOPSr-2 nanocomposite; and (d) TEM image of $\mathrm{SrTiO}_{3}$ nanocubes.

Possibly because of the lower concentration of $\mathrm{SrTiO}_{3}$ nanocubes in nanocomposites (2 wt \% with respect to GO), it is hard to observe $\mathrm{SrTiO}_{3}$ nanoparticles uniformly in the FESEM images of nanocomposites as these particles are embedded within the sheets of PANI-coated GO. Hence, FESEM-mapping and FESEM-EDX might perhaps be an appropriate method to validate the occurrence of $\mathrm{SrTiO}_{3}$ nanoparticles in the matrix of a PANI-coated GO nanocomposite. Figure 2 demonstrates the elemental mapping analysis of GOPSr-2, which reveals that $\mathrm{SrTiO}_{3}$ nanocubes are uniformly present within the matrix of the composite material along with carbon, oxygen, nitrogen, and chlorine. An elemental analysis (Figure S3) displays the occurrence of strontium and titanium, which additionally confirms the formation of $\mathrm{SrTiO}_{3}$ nanocube-doped PANI-coated GO nanocomposites. 

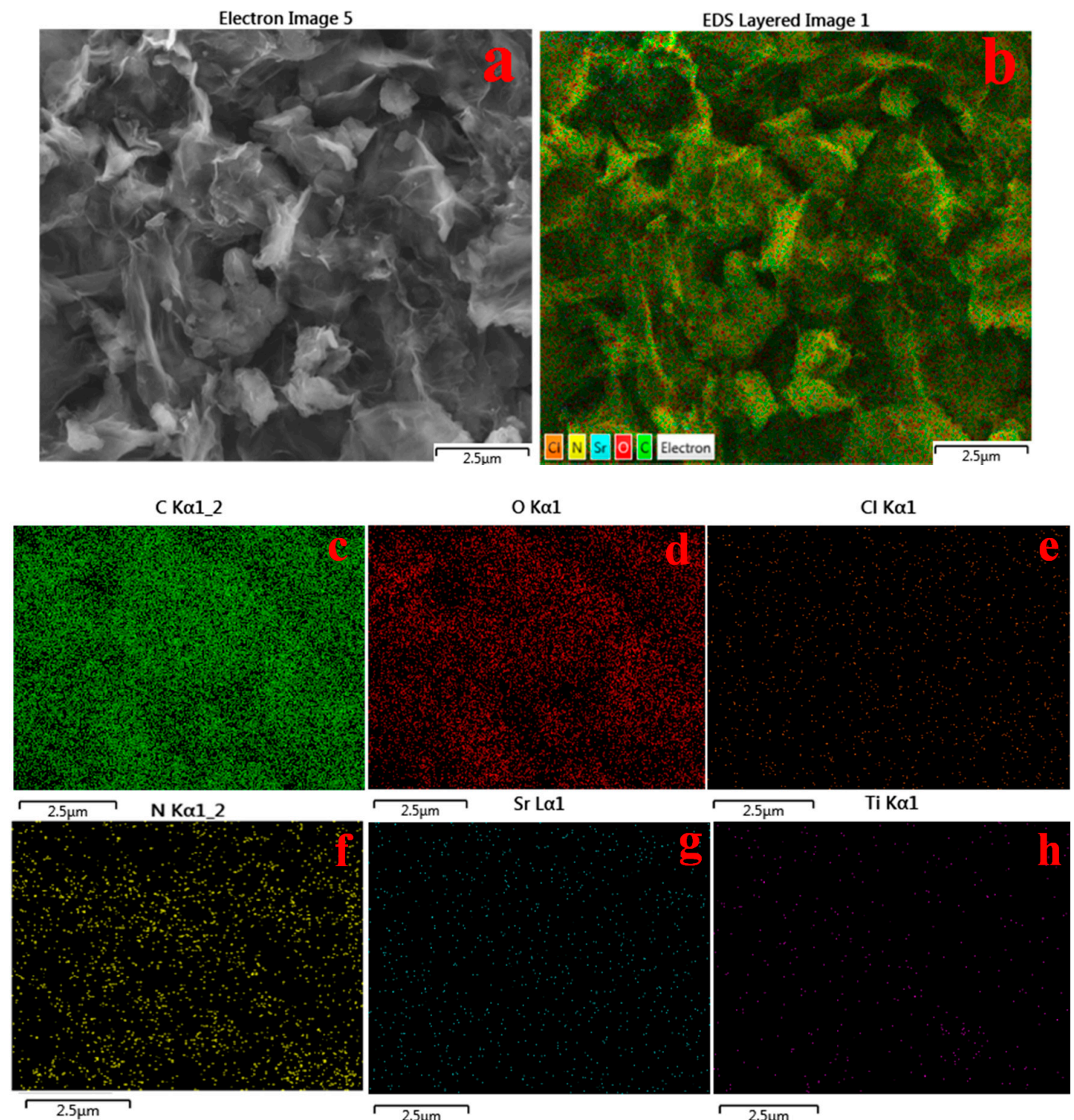

Figure 2. (a) FESEM image and (b) EDX elemental mapping of GOPSr-2 nanocomposite on a Si wafer for the following elements: (c) C; (d) $\mathrm{O}$; (e) $\mathrm{Cl}$; (f) N; (g) Sr; and (h) Ti.

\subsection{FTIR Analysis}

Figure 3 shows the FTIR spectra of PANI, GO, $\mathrm{SrTiO}_{3}, \mathrm{GOPSr}-0$, and GOPSr-2. The IR bands appearing at 1560 and $1480 \mathrm{~cm}^{-1}$ signify the typical C-C stretching of quinoid and benzenoid rings in PANI, respectively. The peak at $1297 \mathrm{~cm}^{-1}$ might be ascribed to $\mathrm{C}-\mathrm{N}$ and $\mathrm{C}=\mathrm{N}$ stretching modes in PANI. IR peaks at 807 and $1127 \mathrm{~cm}^{-1}$ were attributed to out-of-plane $\mathrm{C}-\mathrm{H}$ bending and in-plane $\mathrm{C}-\mathrm{H}$ bending [12,49]. The IR spectrum of GO reveals a broad peak around $3240 \mathrm{~cm}^{-1}$, which may be attributed to the $\mathrm{O}-\mathrm{H}$ stretching vibrations. The IR peaks appearing at 1730, 1613, 1395, and $1219 \mathrm{~cm}^{-1}$ correspond to the $\mathrm{C}=\mathrm{O}$ stretching mode, $\mathrm{sp}^{2}$-hybridized $\mathrm{C}=\mathrm{C}$ stretching and $\mathrm{O}-\mathrm{H}$ bending modes, $\mathrm{C}-\mathrm{OH}$ stretching mode, and $\mathrm{C}-\mathrm{O}-\mathrm{C}$ stretching mode, respectively [50]. Additionally, the peak at $1044 \mathrm{~cm}^{-1}$ could be attributed to the $\mathrm{C}-\mathrm{O}$ vibration due to the epoxy or alkoxy groups [51]. The IR spectrum of $\mathrm{SrTiO}_{3}$ exhibits a band around $3100 \mathrm{~cm}^{-1}$, which may be attributed to the O-H stretching modes in water of crystallization. The IR peaks around $1480 \mathrm{~cm}^{-1}$ could be attributed to the carboxylate group stretching modes, whereas the peaks at 855 and $600 \mathrm{~cm}^{-1}$ are attributed to $\mathrm{TiO}_{6}$ octahedron bending and stretching vibration [12,52] The FTIR spectrum of PANI-coated-GO exhibits the characteristic GO band along with PANI peaks. The PANI peaks appear to be slightly shifted to 
$1568,1482,1257$, and $801 \mathrm{~cm}^{-1}$, as revealed by the IR spectrum of PANI homopolymer. This slight shifting of peaks may be attributed to the change in chemical environment of PANI upon coating over GO, indicating some chemical interaction between PANI chains and GO matrix. The IR spectrum of an $\mathrm{SrTiO}_{3}$-doped nanocomposite, GOPSr-2, exhibits characteristic peaks of both GO and PANI. The band at $1489 \mathrm{~cm}^{-1}$ represents the $\mathrm{SrTiO}_{3}$ carboxylate group stretching mode, which overlaps with $\mathrm{C}-\mathrm{C}$ stretching of quinoid and benzenoid and is slightly shifted. The new peak also appears at $581 \mathrm{~cm}^{-1}$, slightly shifted towards red from its original position $\left(600 \mathrm{~cm}^{-1}\right)$, which might be attributed to $\mathrm{TiO}_{6}$ octahedron bending and the stretching vibration mode. This slight shifting of the band towards red may perhaps be ascribed to some amount of weak van der Waals attraction between the $\mathrm{SrTiO}_{3}$ nanocubes with GO and PANI chains. Hence, the FTIR studies are in good agreement with the reported literature and evidently stipulate the formation of $\mathrm{PANI}, \mathrm{GO}, \mathrm{SrTiO}_{3}$, and $\mathrm{SrTiO}_{3}$-doped PANI-coated GO nanocomposites.

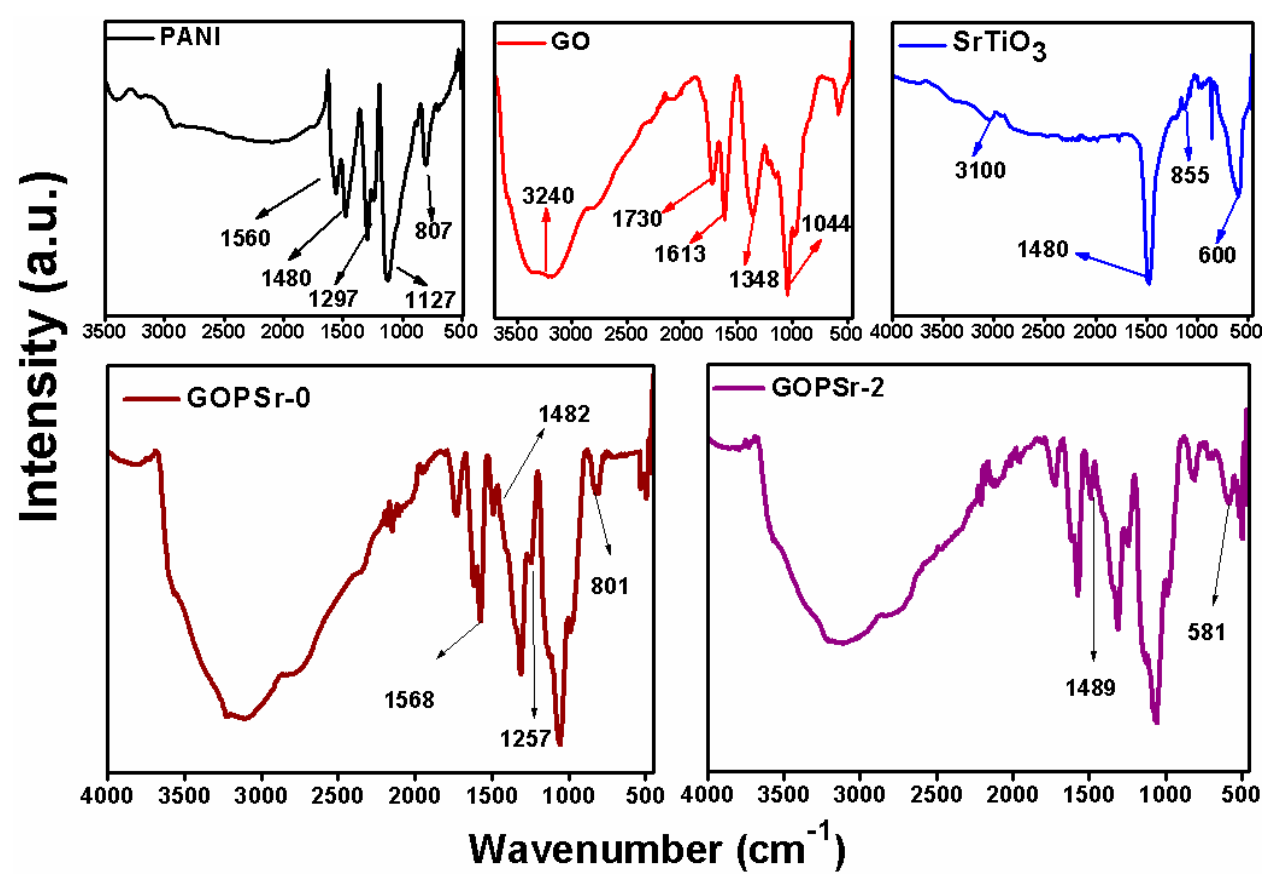

Figure 3. FTIR spectra of PANI, GO, $\mathrm{SrTiO}_{3}, \mathrm{GOPSr}-0$, and GOPSr-2.

\subsection{XRD Analysis}

Figure 4 depicts the wide-angle X-ray diffraction (WAXD) pattern of the powder samples of hydrothermally synthesized $\mathrm{SrTiO}_{3}$ nanocubes, GO, PANI homopolymer, and GOPSr-2. $\mathrm{SrTiO}_{3}$ nanocubes indicated good crystallinity with diffraction peaks corresponding to the (100), (110), (111), (200), (210), (211), (220), (310), (311), and (222) planes of cubic perovskite $\mathrm{SrTiO}_{3}$ structure respectively. These peaks are characteristic of $\mathrm{SrTiO}_{3}$ and can be readily indexed as those of cubic perovskite structure (space group: $\mathrm{Pm} 3 \mathrm{~m}$ ) of $\mathrm{SrTiO}_{3}$ in accordance with JCPDS card No. 35-0734 [12]. The presence of well-defined and very sharp peaks in the XRD pattern of $\mathrm{SrTiO}_{3}$ nanocubes indicates the well-developed crystalline structure. The XRD pattern of the GO illustrated an intense and sharp peak centered at $2 \theta=10.40$, which corresponds to the interplanar spacing of GO sheets. The observed $2 \theta$ for GO could be attributed to the (001) reflection plane, which is usually governed by the process of synthesis and number of layers of water within the interplanar space of GO [34,42]. As is evident from Figure 4, the PANI homopolymer exhibited typical diffraction peaks at $2 \theta$ values of 15.60 , 20.25 , and 25.35, which are the characteristic peaks of conductive PANI. These peaks indicate the polycrystalline nature of the PANI homopolymer [53]. The peaks appearing at angles of $2 \theta$ value of 20.77 and 25.27 epitomise the periodic repetition of benzenoid and quinoid rings in PANI chains [54]. 
As is apparent from the XRD pattern of GOPSr-2, the intense peak of GO has significantly reduced, indicating that the aggregation of GO sheets had been considerably diminished and was abundantly utilized as the substrate by the PANI homopolymer to produce a nanocomposite hybrid material [42]. Characteristic PANI and $\mathrm{SrTiO}_{3}$ diffraction peaks can be seen clearly in the XRD spectrum of GOPSr-2 nanocomposites (marked by asterisks), which prove the uniform occurrence of PANI and $\mathrm{SrTiO}_{3}$ nanocubes in nanocomposites. Therefore, $\mathrm{XRD}$ investigation established the successful synthesis of $\mathrm{SrTiO}_{3}, \mathrm{GO}$, and PANI and the formation of $\mathrm{SrTiO}_{3}$-doped, PANI-coated GO nanocomposite.

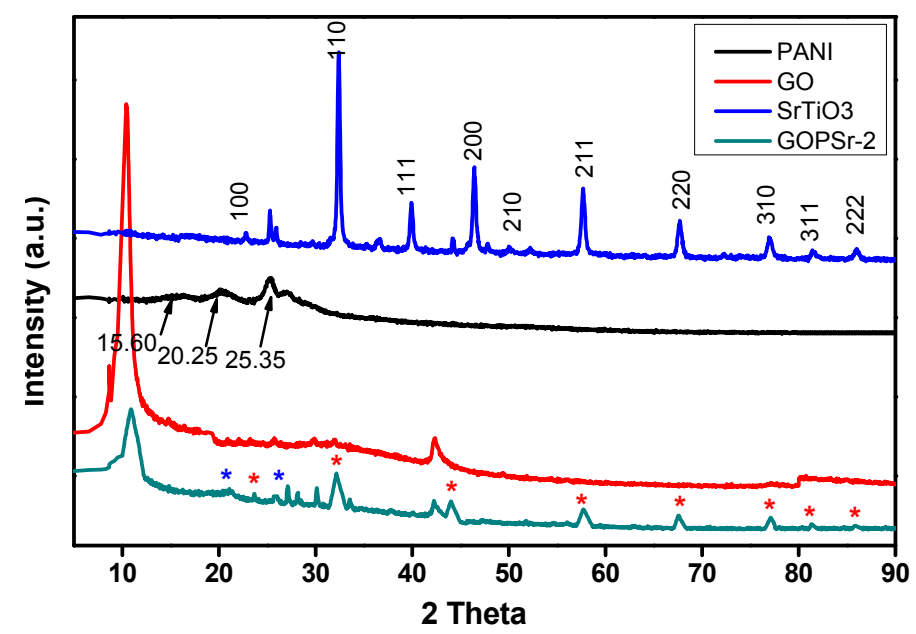

Figure 4. XRD pattern of PANI, GO, SrTiO3, and GOPSr-2.

\subsection{Adsorption Analysis of $M B$ and $M O$}

The aqueous-phase adsorption behaviour of MB and MO dyes was examined in a set of Erlenmeyer flasks by batch process, using a shaker with constant shaking of $180 \mathrm{rpm}$ at ambient temperature, in the presence of $\mathrm{SrTiO}_{3}$ nanocubes, GO, PANI homopolymer, GOPSr-0, GOPSr-1, GOPSr-2, and GOPSr-5 nanocomposites. Figures 5 and 6 show the adsorption behaviour and percentage adsorption of $\mathrm{MB}$ and $\mathrm{MO}$ in the presence of various adsorbents, clearly revealing the efficient adsorption phenomenon taking place between the dyes and adsorbent molecules. The adsorption data demonstrate that $\mathrm{SrTiO}_{3}$ nanocubes, GO, and PANI homopolymers showed lower adsorption efficiencies as compared to the adsorption efficiencies of $\mathrm{SrTiO}_{3}$ nanocubes-doped, polyaniline-coated GO nanocomposites, namely, GOPSr-1, GOPSr-2, and GOPSr-5. As is apparent from Figures $5 b$ and $6 b$, the percentage adsorption for MB depicts the following trend: GOPSr-2 > GOPSr-1 > GOPSr-5 > GOPSr-0 $>$ GO $>$ PANI $>\mathrm{SrTiO}_{3}$, whereas the percentage adsorption for MO illustrates the following trend: GOPSr-2 > GOPSr-5 > GOPSr-1 > GOPSr-0 $>$ PANI $>\mathrm{GO}>\mathrm{SrTiO}_{3}$. Figures $5 \mathrm{a}$ and $6 \mathrm{a}$ exhibit the UV-vis adsorption spectra of the MB and $\mathrm{MO}$ in the presence of different nanocomposites, which indicates that the adsorption efficiency of nanocomposites is greatly enhanced in the presence of $\mathrm{SrTiO}_{3}$ nanocubes as compared to bare GO, PANI, and PANI-coated GO, thereby predicting a synergistic phenomenon between $\mathrm{SrTiO}_{3}$ nanocubes, GO, and PANI. Approximately $99 \%$ of MB and $91 \%$ of MO were removed within a short duration of $30 \mathrm{~min}$, demonstrating the enhanced adsorption efficiency of the GOPSr-2 nanocomposite over $\mathrm{SrTiO}_{3}$ nanocubes, GO, PANI homopolymer, GOPSr-0, GOPSr-1, and GOPSr-5, which exhibited nearly $8 \%$, $57 \%, 18 \%, 78 \%, 87 \%$, and $84 \%$ adsorption for MB but $1.3 \%, 36 \%, 61 \%, 72 \%, 84 \%$ and $89 \%$ adsorption for MO, respectively. Therefore, the adsorption analysis of MB and MO dyes suggests GOPSr-2 nanocomposite is an optimal adsorbent for the efficient removal of carcinogenic MB and MO dyes from aqueous solutions. 

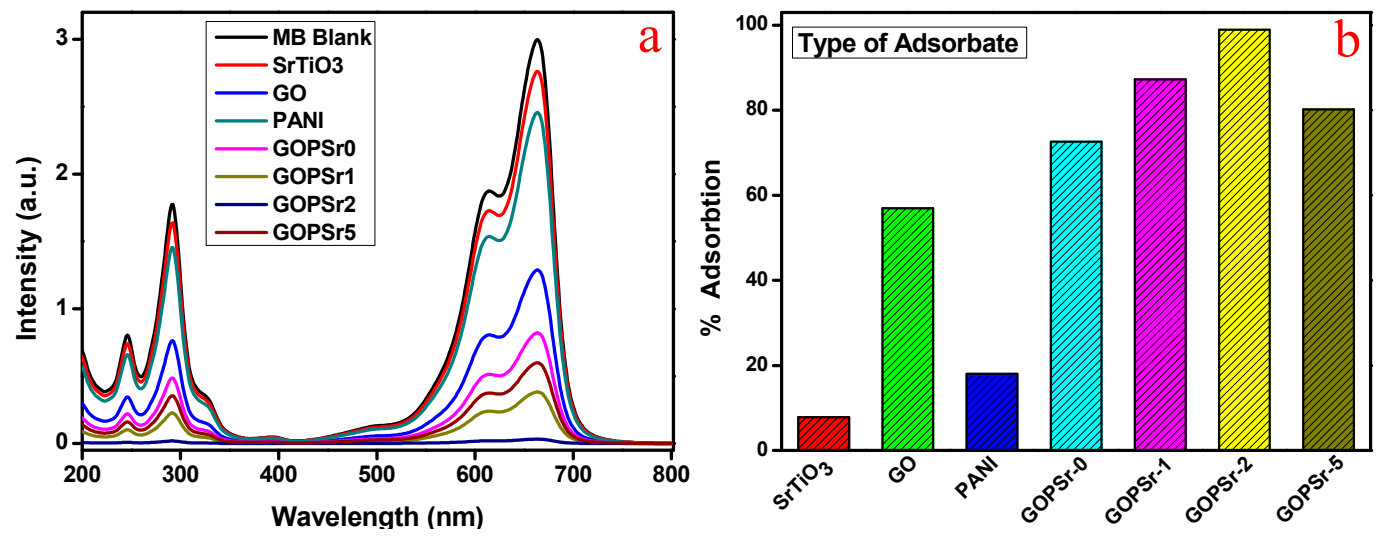

Figure 5. (a) UV-vis absorption spectra of $M B$ aqueous in the presence of various adsorbents and $(\mathbf{b})$ percentage removal of $\mathrm{MB}$ in the presence of various adsorbents (initial MB concentration: $20 \mathrm{mg} \cdot \mathrm{L}^{-1}$; amount of adsorbent: $0.5 \mathrm{mg} \cdot \mathrm{mL}^{-1}$; $\mathrm{pH} 7$; time: $30 \mathrm{~min}$; at room temperature).
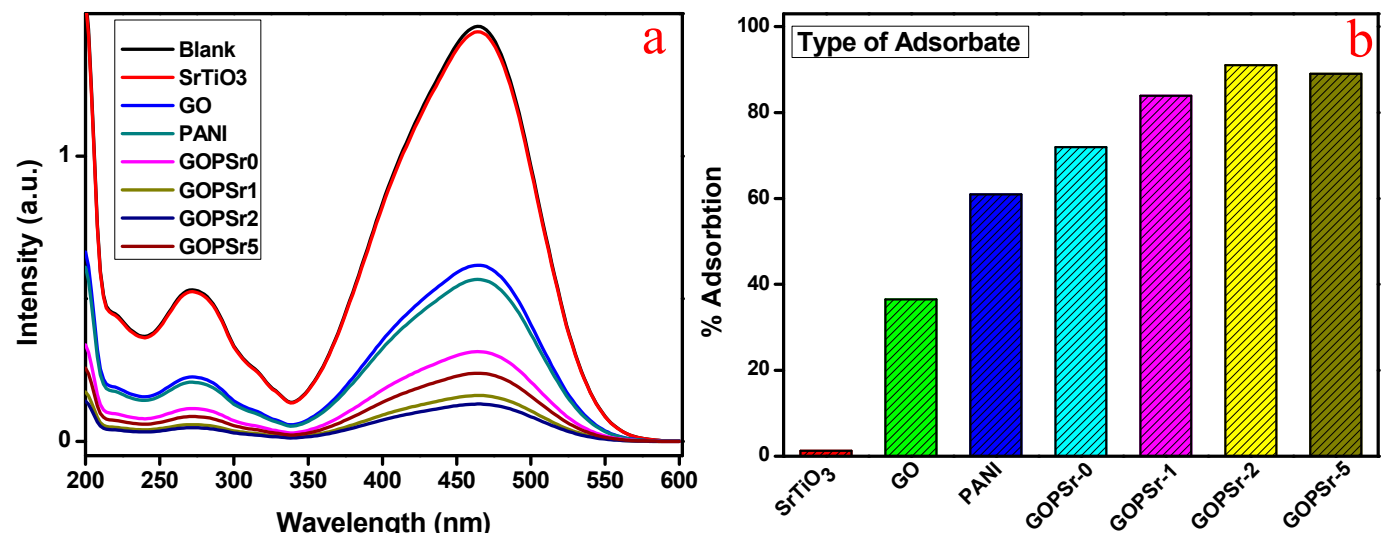

Figure 6. (a) UV-vis absorption spectra of $\mathrm{MO}$ aqueous in the presence of various adsorbents and $(\mathbf{b})$ percentage removal of $\mathrm{MB}$ in the presence of various adsorbents (initial MO concentration: $20 \mathrm{mg} \cdot \mathrm{L}^{-1}$; amount of adsorbent: $0.5 \mathrm{mg} \cdot \mathrm{mL}^{-1}$; $\mathrm{pH} 7$; time: $30 \mathrm{~min}$; at room temperature).

Accumulation of a substance between the liquid-solid interface or gas-solid interface due to physical or chemical associations is termed an adsorption process. With few exceptions, adsorption is usually controlled by physical parameters on most of the adsorbents such as polarity, van der Waals forces, hydrogen bonding, dipole-dipole interaction, $\pi-\pi$ interaction, etc. [49]. Therefore, the design of an adsorbent usually depends on the type of substance to be adsorbed or removed. MB is a cationic dye that can be removed by an adsorbent showing strong affinity towards positively-charged species, whereas $\mathrm{MO}$ is an anionic dye that requires positively polar material for its efficient removal. GO, due to the presence of an $\mathrm{sp}^{2}$ hybridized framework and oxygen-containing functionalities such as hydroxyl and epoxy groups, tends to show enhanced affinity towards cationic species. As is evident from percentage adsorption data, GO alone can adsorb 57\% of MB dye due to its cationic nature, whereas it only removed $36 \%$ of $\mathrm{MO}$, which may perhaps be due to the formation of hydrogen bonding or van der Waal's attractions between MO and GO. On the other hand, polyaniline in its conductive emeraldine salt state possesses a large number of amine $(-\mathrm{N}<)$ and imine $(-\mathrm{N}=)$ functional groups and substantial amounts of positive charges localised over its backbone, making it a suitable candidate for the efficient adsorption of negatively polarised substances. Thus, when PANI alone was used as an adsorbent, it was capable of adsorbing $61 \%$ of $\mathrm{MO}$, while it only adsorbs $18 \%$ of MB. Thus, designing a nanocomposite material comprised of GO coated with conductive chains of PANI may be an alternative material that can adsorb MB as well as MO simultaneously. As illustrated by the percentage adsorption 
results in Figures $5 b$ and $6 b$, PANI-coated GO (GOPSr-0) efficiently adsorbed MB and MO with 78\% and $72 \%$ adsorption efficiency, respectively. However, although the efficiency of PANI-coated GO has enhanced substantially, the overall performance of the nanocomposite was on the lower side. This might be due to agglomeration of disorderly stacked GO sheets, which reduces the effective surface area of the adsorbent and does not allow for efficient adsorption of dye molecules on the surface of the adsorbent. Therefore, in order to segregate the stacked GO layers, $\mathrm{SrTiO}_{3}$ nanocubes were incorporated inside the nanocomposites during the polymerization process (Scheme 1). These $\mathrm{SrTiO}_{3}$ nanocubes adhered to the sheets of GO through $\mathrm{Sr}^{3+}$ ions' interactions with an $\mathrm{sp}^{2}$ hybridized framework of GO. Thus they act as spacers that help in the segregation of GO sheets, thereby enhancing the total effective surface area and increasing the thermal stability of the nanocomposite material, as discussed in the thermal analysis section. Hence, as revealed by adsorption results, $\mathrm{SrTiO}_{3}$ nanocube-doped, polyaniline-coated GO nanocomposites showed highly enhanced adsorption capacity as compared to the neat GO, PANI, and GOPSr-0. The adsorption efficiency increased to $99 \%$ for MB and $91 \%$ for $\mathrm{MO}$ upon doping with $2 \mathrm{wt} \%$ of $\mathrm{SrTiO}_{3}$ with respect to GO. The amount of doping percentage was optimised by varying the concentration of $\mathrm{SrTiO}_{3}$ and it was found that when the percentage of $\mathrm{SrTiO}_{3}$ increased to $5 \%$ there was a slight decrease in adsorption efficiency, which might be due to the agglomeration of $\mathrm{SrTiO}_{3}$ nanocubes at a high doping percentage, implying that GOPSr-2 would be an optimal adsorbent composition.

\subsection{Effect of Adsorbent Dosage}

The effect of adsorbent dosage on percentage removal of $\mathrm{MB}$ and $\mathrm{MO}$ was examined by taking different quantities of GOPSr-2 nanocomposite ranging from 0.25 to $5 \mathrm{mg} \cdot \mathrm{mL}^{-1}$ and investigating the dye adsorption efficiency with an initial concentration of $20 \mathrm{mg} \cdot \mathrm{L}^{-1}, \mathrm{pH} 7$, and ambient temperature for both dyes. Figure 7 depicts the percentage adsorption of MB and MO by GOPSr-2. As is evident from the figure, the percentage adsorption of $\mathrm{MB}$ increases with an increase in the amount of adsorbent from 0.25 to $0.5 \mathrm{mg} \cdot \mathrm{mL}^{-1}$ and becomes constant at a higher dosage. Approximately $85 \%$ of $\mathrm{MB}$ was adsorbed on the surface of the adsorbent when the dosage was $0.25 \mathrm{mg} \cdot \mathrm{mL}^{-1}$ and increased to $99 \%$ on increasing the amount of adsorbent to $0.5 \mathrm{mg} \cdot \mathrm{mL}^{-1}$. On the other hand, for $\mathrm{MO}$, percentage removal increases gradually with the increase in adsorbent dosage from 0.25 to $0.5 \mathrm{mg} \cdot \mathrm{mL}^{-1}$ with $19 \%, 39 \%$, $74 \%, 91 \%$, and $95 \%$ adsorption at an adsorbent dosage of $0.25,0.5,1,2$, and $5 \mathrm{mg} \cdot \mathrm{mL}^{-1}$, respectively. The increase in adsorption efficiency when increasing the amount of adsorbent could be ascribed to the availability of more adsorption sites, and increases in the available effective surface area increased the adsorbent dosage. Moreover, the results reveal that GOPSr-2 has strong affinity toward MB as compared to $\mathrm{MO}$ as percentage removal was found to be $99 \%$ for $\mathrm{MB}$ and $39 \%$ for $\mathrm{MO}$ at an adsorbent dosage of $0.5 \mathrm{mg} \cdot \mathrm{mL}^{-1}$. Therefore, $0.5 \mathrm{and} 2 \mathrm{mg} \cdot \mathrm{mL}^{-1}$ were selected as the optimum dosage for $\mathrm{MB}$ and $\mathrm{MO}$, respectively, for subsequent experiments.
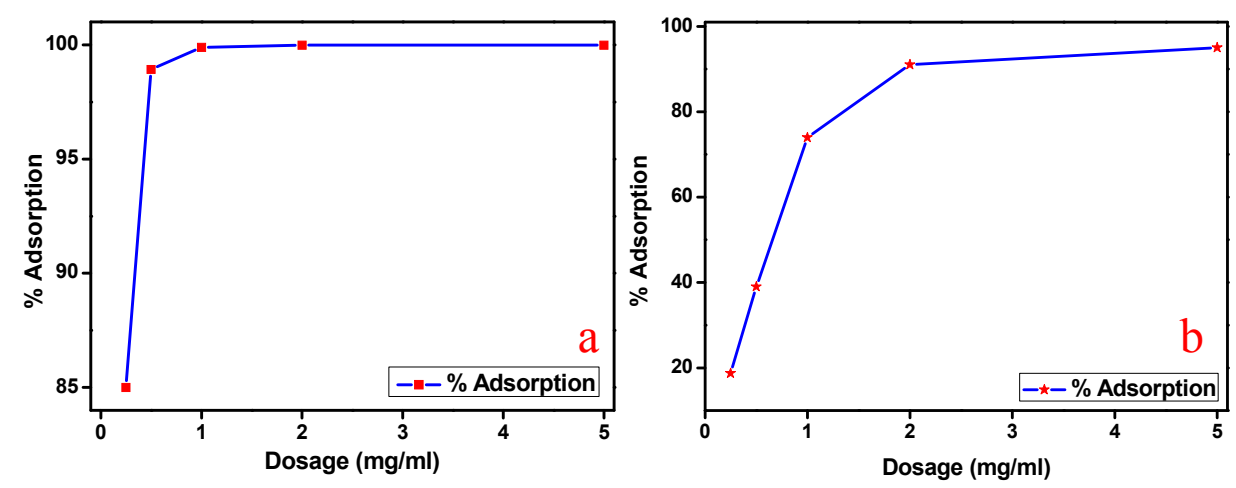

Figure 7. Effect of adsorbent dosage on percentage removal of (a) MB and (b) MO in the presence of GOPSr-2 (initial MB and MO concentration: $20 \mathrm{mg} \cdot \mathrm{L}^{-1}$; $\mathrm{pH} 7$; time: $30 \mathrm{~min}$ at room temperature). 


\subsection{Effect of Contact Time}

Contact time is an important parameter to determine the efficiency of an adsorbent as a rapid rate of adsorption indicates an efficient adsorbent. In order to determine the effect of contact time on the percentage removal of dyes, adsorption was monitored at particular intervals of time ranging from 10 to $60 \mathrm{~min}$ with an initial concentration of $20 \mathrm{mg} \cdot \mathrm{L}^{-1}, \mathrm{pH} 7$, and ambient temperature using an adsorbent dosage of $0.5 \mathrm{mg} \cdot \mathrm{mL}^{-1}$ for $\mathrm{MB}$ and $2 \mathrm{mg} \cdot \mathrm{mL}^{-1}$ for MO. Figure 8 illustrates the effect of contact time on percentage removal of $\mathrm{MB}$ and $\mathrm{MO}$. As is apparent from the figure, approximately $99 \%$ of MB was adsorbed on the surface of GOPSr- 2 within $10 \mathrm{~min}$, indicating rapid and efficient adsorption. Alternatively, the adsorption of MO increases steadily with the increase in time and reaches equilibrium at $40 \mathrm{~min}$ of contact time, with approximately $94 \%$ of dye adsorption. Thus, based upon the following experiment, 10 and $40 \mathrm{~min}$ were selected as the optimized contact times for further experiments.
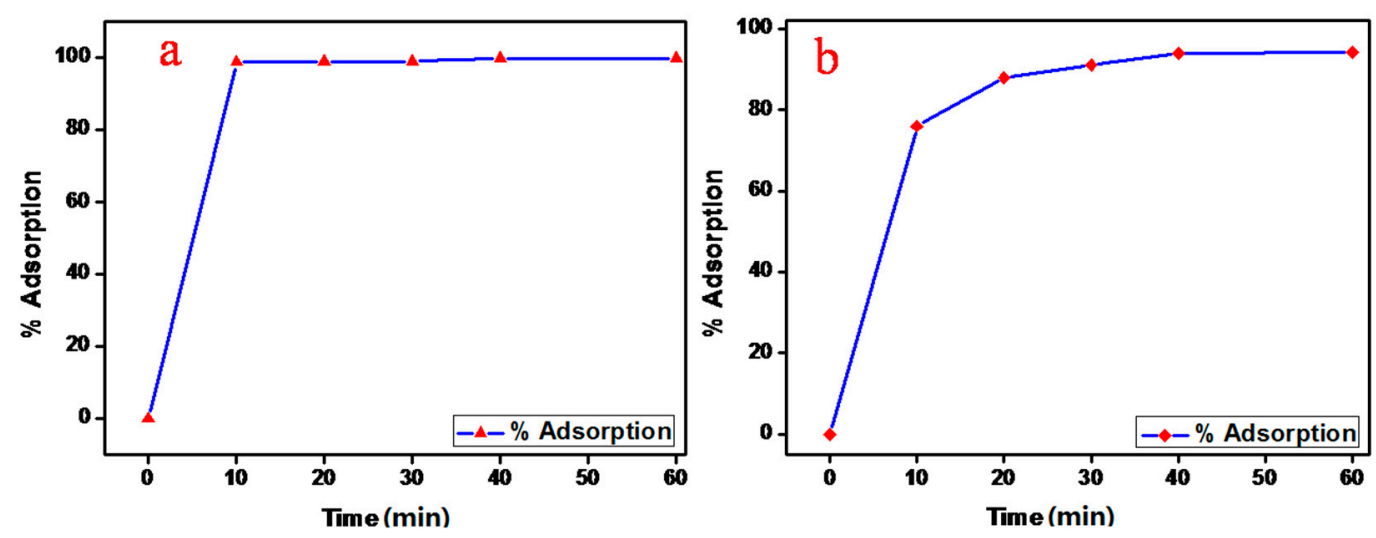

Figure 8. Effect of time on percentage removal of (a) MB and (b) MO in the presence of GOPSr-2 (initial $\mathrm{MB}$ and $\mathrm{MO}$ concentration: $20 \mathrm{mg} \cdot \mathrm{L}^{-1}$; amount of adsorbent $(\mathrm{MB}): 0.5 \mathrm{mg} \cdot \mathrm{mL}^{-1},(\mathrm{MO})$ : $2 \mathrm{mg} \cdot \mathrm{mL}^{-1} ; \mathrm{pH}(\mathrm{MB}): 9.5$, (MO): 7; at room temperature).

\subsection{Effect of $p H$}

Amongst the various physical parameters, the most significant factor influencing the efficiency of an adsorbent in wastewater treatment is the $\mathrm{pH}$ of the solution. The effectiveness of adsorption is reliant on the $\mathrm{pH}$ of the medium, since diversity in $\mathrm{pH}$ prompts variations in the surface properties of the adsorbent and in the degree of ionization of the dye molecules [7]. Hence, the effect of solution $\mathrm{pH}$ on the percentage removal of MB and MO dyes was investigated using GOPSr- 2 as an adsorbent at a pH range from 4.5 to 9.5, adjusted by the addition of $0.1 \mathrm{~N} \mathrm{HCl}$ or $0.1 \mathrm{~N} \mathrm{NaOH}$ at ambient temperature. As depicted by Figure 9, the adsorption of MB does not show any significant change from $\mathrm{pH} 4.5$ until 9.5, thereby indicating that GOPSr-2 is an excellent adsorbent material that works at a wide range of $\mathrm{pH}$ values. However, $\mathrm{MO}$ adsorption on GOPSr-2 seems to be $\mathrm{pH}$-dependent, with maximum adsorption attained at $\mathrm{pH}$ 7. This might be explained on the basis of a $\mathrm{pKa}$ value of $\mathrm{MO}$ that is around 3.4. Therefore, if the $\mathrm{pKa}$ is more than the $\mathrm{pH}$ of $\mathrm{MO}$, which predominantly exists in anionic form, it gets protonated. Additionally, in an acidic environment PANI tends to develop a positive charge on its conductive backbone due to the presence of basic (imine and amine) groups. Thus, at acidic $\mathrm{pH}$ values a significant amount of electrostatic repulsion occurred between the positively-charged PANI backbone and protonated MO molecules, which tends to decrease the adsorption efficiency of GOPSr-2 for $\mathrm{MO}$ adsorption [55]. At $\mathrm{pH}$ values above 7, adsorption behaviour illustrates a gradual decrease up to $\mathrm{pH}$ 9.5, which might be owing to the competitive adsorption of hydroxyl ions on imine and amine groups, resulting in a decline in $\mathrm{MO}$ adsorption. Thus, for both dyes, the maximum adsorption was achieved at a neutral $\mathrm{pH}(7)$, with approximately $98 \%$ adsorption for $\mathrm{MB}$ and $91 \%$ adsorption for $\mathrm{MO}$. 

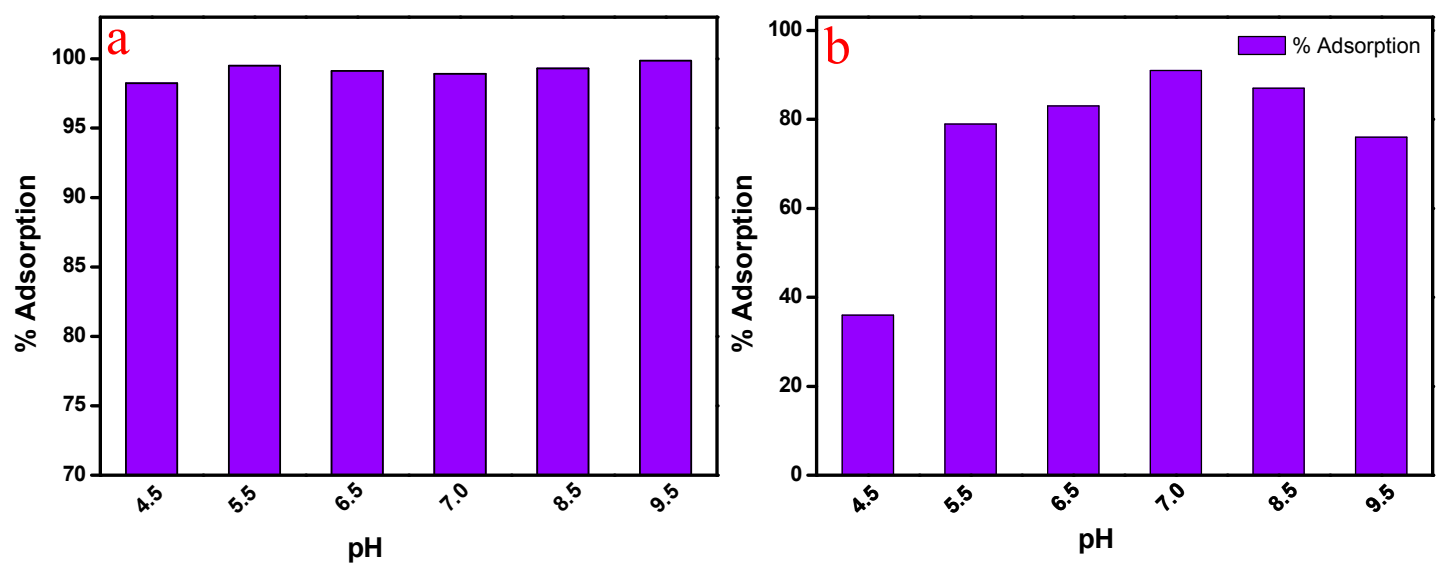

Figure 9. Effect of $\mathrm{pH}$ on percentage removal of (a) MB and (b) MO in the presence of GOPSr-2 (initial $\mathrm{MB}$ and MO concentration: $20 \mathrm{mg} \cdot \mathrm{L}^{-1}$; amount of adsorbent (MB): $0.5 \mathrm{mg} \cdot \mathrm{mL}^{-1}$, (MO): $2 \mathrm{mg} \cdot \mathrm{mL}^{-1}$; time: $30 \mathrm{~min}$ at room temperature).

\subsection{Effect of $\mathrm{NaCl}$ Concentration}

One of the most significant features of adsorption investigations, reported by many researchers, is the effect of salt concentration on the percentage removal of dyes from industrial dye waste water. Since commercial dye waste water generally contains high salt contents, it becomes crucial to study the effect of salt concentration on the percentage removal of $\mathrm{MB}$ and $\mathrm{MO}$ from aqueous solutions using GOPSr-2 nanocomposites. Figure 10 illustrates the effect of $\mathrm{NaCl}$ with variable concentrations ranging from 10 to $50 \mathrm{~g} \cdot \mathrm{L}^{-1}$ on MB and MO removal percentage by GOPSr-2. As is apparent from Figure 10, the adsorption efficacy of nanocomposite is considerably influenced by the presence of $\mathrm{NaCl}$. The adsorption capability of the nanocomposite declines with the increase in $\mathrm{NaCl}$ concentration from 10 to $50 \mathrm{~g} \cdot \mathrm{L}^{-1}$. The percentage removal decreases from $99 \%$ to $89.60 \%$ and $95 \%$ to $81.75 \%$ with an increase in concentration of $\mathrm{NaCl}$ of $50 \mathrm{~g} \cdot \mathrm{L}^{-1}$ for $\mathrm{MB}$ and $\mathrm{MO}$, respectively. This reduction in adsorption efficiency may be attributed to the neutralization of the surface charge of the adsorbent by electrolyte ions that compete with dye molecules for adsorption on the surface of the nanocomposite. Nevertheless, this decline in adsorption efficiency is not very high, which implies that GOPSr-2 can effectively remove MB and $\mathrm{MO}$ from an aqueous solution even in the presence of a high concentration of salt.

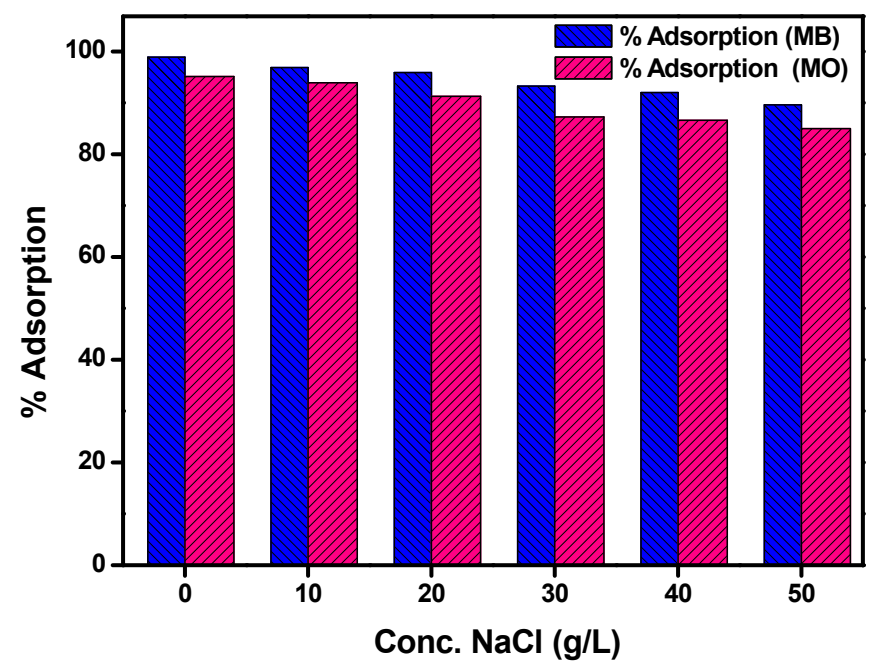

Figure 10. Effect of $\mathrm{NaCl}$ conc. on percentage removal of $\mathrm{MB}$ and $\mathrm{MO}$ in the presence of GOPSr-2 (initial $\mathrm{MB}$ and $\mathrm{MO}$ concentration: $20 \mathrm{mg} \cdot \mathrm{L}^{-1}$; amount of adsorbent $(\mathrm{MB}): 0.5 \mathrm{mg} \cdot \mathrm{mL}^{-1}$, (MO): $2 \mathrm{mg} \cdot \mathrm{mL}^{-1} ; \mathrm{pH}(\mathrm{MB}): 9.5$, (MO): 7; at room temperature). 


\subsection{Reusability Studies}

The stability and reusability of the adsorbent are considered to be important factors for practical application and need to be thoroughly examined. Hence, reusability investigations of GOPSr-2 were conducted to examine the effect of nanocomposites on adsorption capacity after repeated usage cycles. Figure 11 illustrates the reusability of GOPSr-2 for the adsorption of MB and MO up to five cycles. After the first cycle, the adsorbent was recovered via centrifugation and filtration, followed by thorough washing with deionised water, drying at $80^{\circ} \mathrm{C}$ in a vacuum oven for two hours, and successively employing it as an adsorbent for additional cycles so as to study their adsorptive efficiencies. As is apparent from Figure 11, there is a marginal decrease in the adsorption efficiency of the adsorbent after each repeated cycle. The percentage removal for MB was found to be $96.50 \%$, $92.75 \%, 87.20 \%$, and $84.15 \%$ and for MO was found to be $94.20 \%, 91.35 \%, 85.90 \%$, and $81.75 \%$ for the second, third, fourth, and fifth cycles, respectively. This decrease in efficiency could be due to the fact that substantial unavoidable weight loss occurred during the recovery and purification of the adsorbent, which contributes to a decrease in adsorption efficiency in each repeated cycle as well as a reduction in active available sites due to some adsorbed dye molecules. Figure S4 represents the FESEM image of GOPSr-2 after the fifth reusability cycle. As is evident from Figure S4, there is no apparent change in the morphology of the nanocomposite, which indicates the high stability of GOPSr-2 even after repeated usage. However, the investigation clearly reveals that approximately $84 \%$ of MB and $82 \%$ of MO can be removed even in the fifth cycle, signifying the high stability and reusability efficiency of the GOPSr-2 nanocomposite.

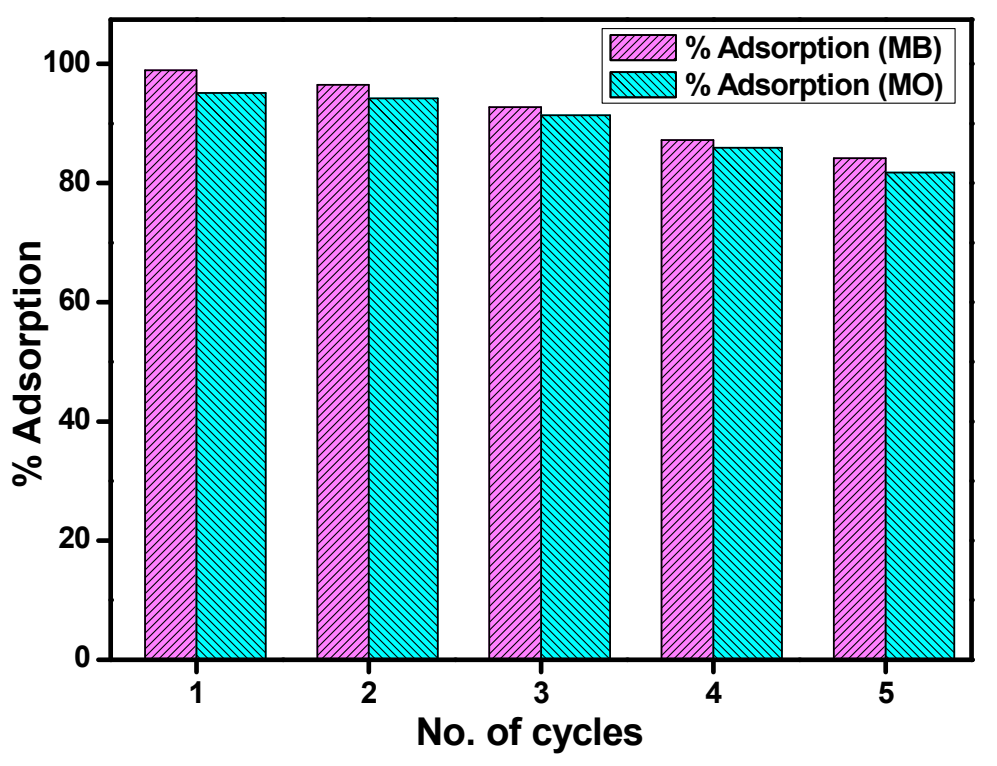

Figure 11. Reproducibility cycles of GOPSr-2 for MB and MO adsorption (initial $\mathrm{MB}$ and $\mathrm{MO}$ concentration: $20 \mathrm{mg} \cdot \mathrm{L}^{-1}$; amount of adsorbent (MB): $0.5 \mathrm{mg} \cdot \mathrm{mL}^{-1}$, (MO): $2 \mathrm{mg} \cdot \mathrm{mL}^{-1}$; $\mathrm{pH}(\mathrm{MB})$ : 9.5, (MO): 7; at room temperature).

\subsection{Proposed Mechanism}

Scheme 2 illustrates the schematic representation of the proposed adsorption mechanism of MB and MO by GOPSr-2. The adsorption mechanism can be explained as follows:

Since $\mathrm{MB}$ and $\mathrm{MO}$ are cationic and anionic in nature, electrostatic attraction is one of the major factors that enhances the efficient removal of charged dyes. GO contains hydroxyl and epoxy groups, along with an $\mathrm{sp}^{2}$ hybridized framework that makes it overall negatively polarized, thereby forming strong electrostatic interaction with $\mathrm{MB}$ for its efficient removal. On the other hand, due to the presence of a positively-charged polymeric backbone, PANI contributes to the efficient removal of MO through 
electrostatic attraction (this has been comprehensively discussed in Section 3.5). Furthermore, since the surface of GOPSr-2 contains hydroxyl and carboxyl functional groups on its surface, whereas MB and $\mathrm{MO}$ contain nitrogen atoms within their structure, the lone pair of nitrogen atoms forms intermolecular H-bonding with these functional groups, thereby aiding in their efficient removal from the aqueous solution. Moreover, $\mathrm{MB}$ and $\mathrm{MO}$ dyes are ideally planar molecules that can be easily adsorbed on the surface of GOPSr-2 via $\pi-\pi$ interaction between the aromatic backbone of the dye and the aromatic skeleton of the GOPSr-2 adsorbent. Therefore, all these physical forces, namely, electrostatic interaction, intermolecular $\mathrm{H}$-bonding, and $\pi-\pi$ interaction, work synergistically and lead to the efficient removal of dyes from aqueous solutions within a short period of time.

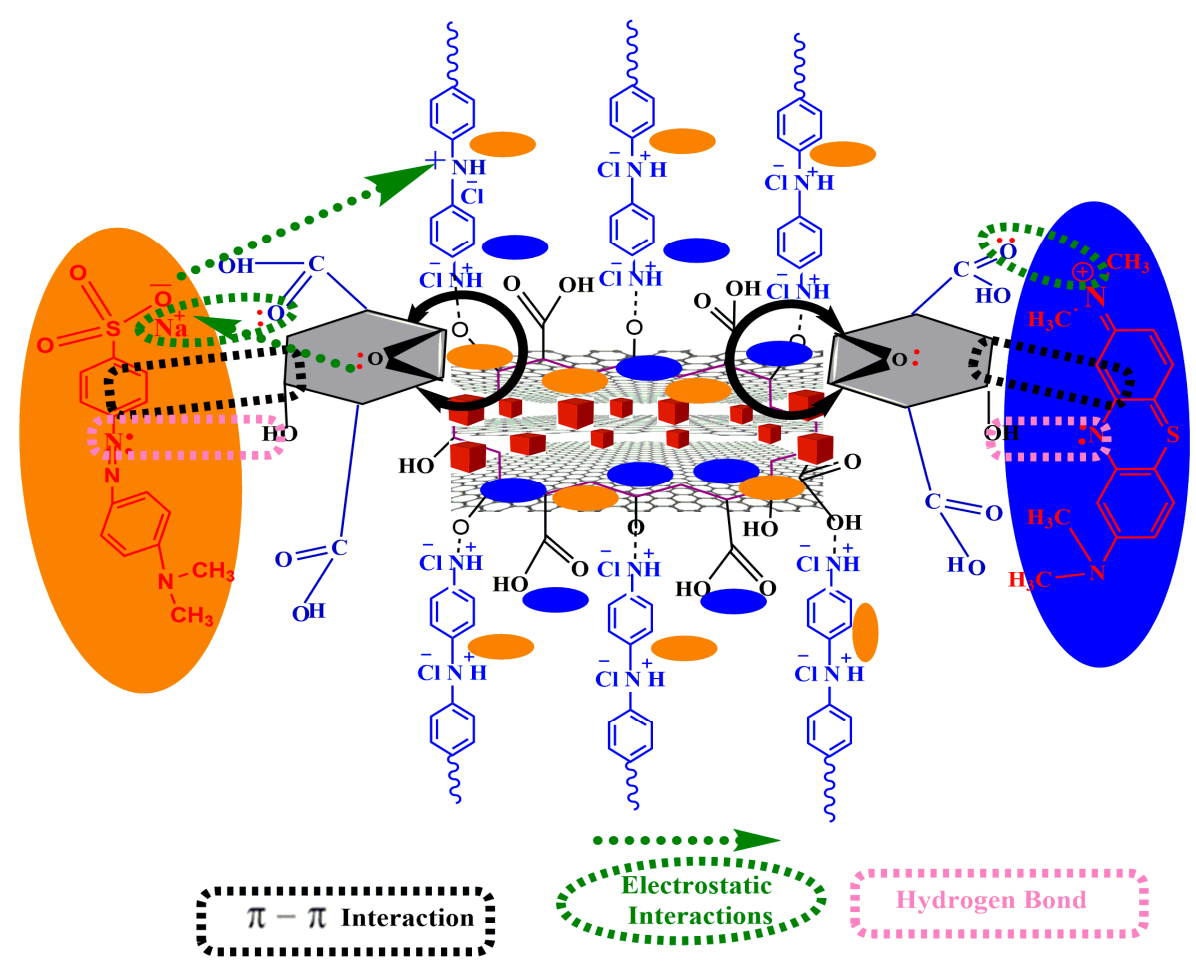

Scheme 2. Proposed mechanism for the adsorption of MB and MO on the surface of GOPSr-2.

\section{Conclusions}

Polyaniline-coated graphene oxide doped with $\mathrm{SrTiO}_{3}$ nanocube nanocomposites has been successfully synthesised via a simple in situ oxidative polymerisation technique. PANI was successfully coated onto the $\mathrm{GO}$ sheets and $\mathrm{SrTiO}_{3}$ nanocubes were successfully incorporated within the matrix of nanocomposite. The surface morphology of GO was completely transformed from aggregated sheets to more segregated ones upon incorporation of PANI and $\mathrm{SrTiO}_{3}$ nanocubes, as depicted by FESEM analysis. The synthesised nanocomposites exhibited greater adsorption efficiencies as compared to bare GO and PANI homopolymers, suggesting a synergistic phenomenon taking place between the polymer chain, graphene sheets, and cubic nanoparticles. The removal efficiency of MB was found to be largely unaffected by a change in $\mathrm{pH}$, whereas $\mathrm{MO}$ was found to be $\mathrm{pH}$-dependent with maximum dye adsorption at $\mathrm{pH}$ 7. No significant decrease in adsorption capacity was observed even at high salt concentrations. Furthermore, the synthetic methodology proposed here may be used for the synthesis of numerous $\mathrm{SrTiO}_{3}$ nanocube-doped GO nanocomposites materials utilising other conducting polymers, which may help address present concerns about environmental pollution.

Supplementary Materials: The following are available online at www.mdpi.com/2073-4360/8/8/305/s1, Figure S1: FESEM images of (a) and (b) GOPSr-2 nanocomposite at different magnifications, Figure S2: TEM 
images GOPSr-2 nanocomposite at different magnifications, Figure S3: EDX spectrum of GOPSr-2 nanocomposite, Figure S4: FESEM image of GOPSr-2 nanocomposite after fifth reusability cycle.

Acknowledgments: The authors would like to thank the University of Malaya (UM) and the Ministry of Higher Education (MOHE) for the research facilities and financial support through Fundamental Research Grant Scheme, FRGS (FP031-2014B), University Malaya Research Grant, UMRG (RP006A-13SUS) and High Impact Research grant (HIR/MOHE/SC/F0031).

Author Contributions: All authors contributed to this study. Syed Shahabuddin designed, executed the experiments and composed this paper. Norazilawati Muhamad Sarih edited the paper and gave final approval of the version to be submitted. Sharifah Mohamad, Muhammad Afzal Kamboh, and Hamid Rashidi Nodeh contributed to the results analysis; Sharifah Mohamad contributed the analysis tools. All authors participated in the discussion and commented on the paper.

Conflicts of Interest: The authors declare no conflict of interest.

\section{References}

1. Sen, T.K.; Afroze, S.; Ang, H. Equilibrium, kinetics and mechanism of removal of methylene blue from aqueous solution by adsorption onto pine cone biomass of pinus radiata. Water Air Soil Pollut. 2011, 218, 499-515. [CrossRef]

2. Sokolowska-Gajda, J.; Freeman, H.S.; Reife, A. Synthetic dyes based on environmental considerations. Part 2: Iron complexes formazan dyes. Dyes Pigment. 1996, 30, 1-20. [CrossRef]

3. Ivanov, K.; Gruber, E.; Schempp, W.; Kirov, D. Possibilities of using zeolite as filler and carrier for dyestuffs in paper. Papier 1996, 50, 7-8.

4. Kabdaşli, I.; Tünay, O.; Orhon, D. Wastewater control and management in a leather tanning district. Water Sci. Technol. 1999, 40, 261-267. [CrossRef]

5. Bensalah, N.; Alfaro, M.Q.; Martínez-Huitle, C. Electrochemical treatment of synthetic wastewaters containing alphazurine a dye. Chem. Eng. J. 2009, 149, 348-352. [CrossRef]

6. Dawood, S.; Sen, T.K.; Phan, C. Synthesis and characterisation of novel-activated carbon from waste biomass pine cone and its application in the removal of congo red dye from aqueous solution by adsorption. Water Air Soil Pollut. 2014, 225, 1-16. [CrossRef]

7. Yagub, M.T.; Sen, T.K.; Afroze, S.; Ang, H.M. Dye and its removal from aqueous solution by adsorption: A review. Adv. Colloid Interface Sci. 2014, 209, 172-184. [CrossRef] [PubMed]

8. Chequer, F.M.D.; de Oliveira, D.P.; Ferraz, E.R.A.; de Oliveira, G.A.R.; Cardoso, J.C.; Zanoni, M.V.B. Textile Dyes: Dyeing Process and Environmental Impact; InTech Open Access Publisher: Rijeka, Croatia, 2013.

9. Couto, S.R. Dye removal by immobilised fungi. Biotechnol. Adv. 2009, 27, 227-235. [CrossRef] [PubMed]

10. Shahabuddin, S.; Sarih, N.M.; Ismail, F.H.; Shahid, M.M.; Huang, N.M. Synthesis of chitosan grafted-polyaniline $/ \mathrm{Co}_{3} \mathrm{O}_{4}$ nanocube nanocomposites and their photocatalytic activity toward methylene blue dye degradation. RSC Adv. 2015, 5, 83857-83867. [CrossRef]

11. Aksu, Z. Application of biosorption for the removal of organic pollutants: A review. Process Biochem. 2005, 40, 997-1026. [CrossRef]

12. Shahabuddin, S.; Muhamad Sarih, N.; Mohamad, S.; Joon Ching, J. SrtiO 3 nanocube-doped polyaniline nanocomposites with enhanced photocatalytic degradation of methylene blue under visible light. Polymers 2016. [CrossRef]

13. Papić, S.; Koprivanac, N.; Božić, A.L.; Meteš, A. Removal of some reactive dyes from synthetic wastewater by combined Al (III) coagulation/carbon adsorption process. Dyes Pigment. 2004, 62, 291-298. [CrossRef]

14. Gonzalez-Gutierrez, L.V.; Escamilla-Silva, E.M. Reactive red azo dye degradation in a UASB bioreactor: Mechanism and kinetics. Eng. Life Sci. 2009, 9, 311-316. [CrossRef]

15. Zou, H.; Ma, W.; Wang, Y. A novel process of dye wastewater treatment by linking advanced chemical oxidation with biological oxidation. Arch. Environ. Prot. 2015, 41, 33-39. [CrossRef]

16. Akbari, A.; Remigy, J.; Aptel, P. Treatment of textile dye effluent using a polyamide-based nanofiltration membrane. Chem. Eng. Processing 2002, 41, 601-609. [CrossRef]

17. Haldorai, Y.; Shim, J.-J. An efficient removal of methyl orange dye from aqueous solution by adsorption onto chitosan/MgO composite: A novel reusable adsorbent. Appl. Surf. Sci. 2014, 292, 447-453. [CrossRef] 
18. Mahto, T.K.; Chandra, S.; Haldar, C.; Sahu, S.K. Kinetic and thermodynamic study of polyaniline functionalized magnetic mesoporous silica for magnetic field guided dye adsorption. RSC Adv. 2015, 5, 47909-47919. [CrossRef]

19. Hosseini Koupale, E.; Alavi Moghaddam, M.; Hashemi, S. Successful treatment of high azo dye concentration wastewater using combined anaerobic/aerobic granular activated carbon-sequencing batch biofilm reactor (GAC-SBBR): Simultaneous adsorption and biodegradation processes. Water Sci. Technol. 2013, 67, 1816-1821. [CrossRef] [PubMed]

20. Hu, J.; Song, Z.; Chen, L.; Yang, H.; Li, J.; Richards, R. Adsorption properties of MgO (111) nanoplates for the dye pollutants from wastewater. J. Chem. Eng. Data 2010, 55, 3742-3748. [CrossRef]

21. Valix, M.; Cheung, W.H.; McKay, G. Sulfur fixation on bagasse activated carbon by chemical treatment and its effect on acid dye adsorption. Adsorption 2009, 15, 453-459. [CrossRef]

22. Ma, J.; Yu, F.; Zhou, L.; Jin, L.; Yang, M.; Luan, J.; Tang, Y.; Fan, H.; Yuan, Z.; Chen, J. Enhanced adsorptive removal of methyl orange and methylene blue from aqueous solution by alkali-activated multiwalled carbon nanotubes. ACS Appl. Mater. Interfaces 2012, 4, 5749-5760. [CrossRef] [PubMed]

23. Bairi, V.G.; Bourdo, S.E.; Sacre, N.; Nair, D.; Berry, B.C.; Biris, A.S.; Viswanathan, T. Ammonia gas sensing behavior of tanninsulfonic acid doped polyaniline- $\mathrm{TiO}_{2}$ composite. Sensors 2015, 15, 26415-26429. [CrossRef] [PubMed]

24. Sengodu, P.; Deshmukh, A.D. Conducting polymers and their inorganic composites for advanced Li-ion batteries: A review. RSC Adv. 2015, 5, 42109-42130. [CrossRef]

25. Hu, Z.; Zu, L.; Jiang, Y.; Lian, H.; Liu, Y.; Li, Z.; Chen, F.; Wang, X.; Cui, X. High specific capacitance of polyaniline/mesoporous manganese dioxide composite using $\mathrm{Ki}^{-} \mathrm{H}_{2} \mathrm{SO}_{4}$ electrolyte. Polymers 2015, 7, 1939-1953. [CrossRef]

26. Bhadra, S.; Khastgir, D.; Singha, N.K.; Lee, J.H. Progress in preparation, processing and applications of polyaniline. Prog. Polym. Sci. 2009, 34, 783-810. [CrossRef]

27. Shih, H.-K.; Chen, Y.-H.; Chu, Y.-L.; Cheng, C.-C.; Chang, F.-C.; Zhu, C.-Y.; Kuo, S.-W. Photo-crosslinking of pendent uracil units provides supramolecular hole injection/transport conducting polymers for highly efficient light-emitting diodes. Polymers 2015, 7, 804-818. [CrossRef]

28. Baharin, S.N.A.; Muhamad Sarih, N.; Mohamad, S.; Shahabuddin, S.; Sulaiman, K.; Ma'amor, A. Removal of endocrine disruptor di-(2-ethylhexyl)phthalate by modified polythiophene-coated magnetic nanoparticles: Characterization, adsorption isotherm, kinetic study, thermodynamics. RSC Adv. 2016, 6, 44655-44667. [CrossRef]

29. Boeva, Z.A.; Sergeyev, V. Polyaniline: Synthesis, properties, and application. Polym. Sci. Ser. C 2014, 56, 144-153. [CrossRef]

30. Tiwari, A. Gum arabic-graft-polyaniline: An electrically active redox biomaterial for sensor applications. J. Macromol. Sci Part A 2007, 44, 735-745. [CrossRef]

31. Zhao, X.; Lv, L.; Pan, B.; Zhang, W.; Zhang, S.; Zhang, Q. Polymer-supported nanocomposites for environmental application: A review. Chem. Eng. J. 2011, 170, 381-394. [CrossRef]

32. Zhu, Y.; Murali, S.; Cai, W.; Li, X.; Suk, J.W.; Potts, J.R.; Ruoff, R.S. Graphene and graphene oxide: Synthesis, properties, and applications. Adv. Mater. 2010, 22, 3906-3924. [CrossRef] [PubMed]

33. Golsheikh, A.M.; Lim, H.N.; Zakaria, R.; Huang, N.M. Sonochemical synthesis of reduced graphene oxide uniformly decorated with hierarchical zns nanospheres and its enhanced photocatalytic activities. RSC Adv. 2015, 5, 12726-12735. [CrossRef]

34. Zhang, K.; Zhang, L.L.; Zhao, X.; Wu, J. Graphene/polyaniline nanofiber composites as supercapacitor electrodes. Chem. Mater. 2010, 22, 1392-1401. [CrossRef]

35. Huang, X.; Qi, X.; Boey, F.; Zhang, H. Graphene-based composites. Chem. Soc. Rev. 2012, 41, 666-686. [CrossRef] [PubMed]

36. Stoller, M.D.; Park, S.; Zhu, Y.; An, J.; Ruoff, R.S. Graphene-based ultracapacitors. Nano Lett. 2008, 8, 3498-3502. [CrossRef] [PubMed]

37. Kuilla, T.; Bhadra, S.; Yao, D.; Kim, N.H.; Bose, S.; Lee, J.H. Recent advances in graphene based polymer composites. Prog. Polym. Sci. 2010, 35, 1350-1375. [CrossRef]

38. Jahan, M.; Bao, Q.; Yang, J.-X.; Loh, K.P. Structure-directing role of graphene in the synthesis of metal-organic framework nanowire. J. Am. Chem. Soc. 2010, 132, 14487-14495. [CrossRef] [PubMed] 
39. Petit, C.; Bandosz, T.J. Enhanced adsorption of ammonia on metal-organic framework/graphite oxide composites: Analysis of surface interactions. Adv. Funct. Mater. 2010, 20, 111-118. [CrossRef]

40. Jasuja, K.; Berry, V. Implantation and growth of dendritic gold nanostructures on graphene derivatives: Electrical property tailoring and Raman enhancement. Acs Nano 2009, 3, 2358-2366. [CrossRef] [PubMed]

41. Wang, H.; Hao, Q.; Yang, X.; Lu, L.; Wang, X. Graphene oxide doped polyaniline for supercapacitors. Electrochem. Commun. 2009, 11, 1158-1161. [CrossRef]

42. Kumar, N.A.; Choi, H.-J.; Shin, Y.R.; Chang, D.W.; Dai, L.; Baek, J.-B. Polyaniline-grafted reduced graphene oxide for efficient electrochemical supercapacitors. ACS Nano 2012, 6, 1715-1723. [CrossRef] [PubMed]

43. Yong, Y.-C.; Dong, X.-C.; Chan-Park, M.B.; Song, H.; Chen, P. Macroporous and monolithic anode based on polyaniline hybridized three-dimensional graphene for high-performance microbial fuel cells. ACS Nano 2012, 6, 2394-2400. [CrossRef] [PubMed]

44. Shahabuddin, S.; Sarih, N.M.; Mohamad, S.; Atika Baharin, S.N. Synthesis and characterization of $\mathrm{Co}_{3} \mathrm{O}_{4}$ nanocube-doped polyaniline nanocomposites with enhanced methyl orange adsorption from aqueous solution. RSC Adv. 2016, 6, 43388-43400. [CrossRef]

45. Zhang, Z.; Kong, J. Novel magnetic $\mathrm{Fe}_{3} \mathrm{O}_{4} @ \mathrm{C}$ nanoparticles as adsorbents for removal of organic dyes from aqueous solution. J. Hazard. Mater. 2011, 193, 325-329. [CrossRef] [PubMed]

46. Nassar, M.Y.; Ahmed, I.S. Template-free hydrothermal derived cobalt oxide nanopowders: Synthesis, characterization, and removal of organic dyes. Mater. Res. Bull. 2012, 47, 2638-2645. [CrossRef]

47. Jiao, F.; Frei, H. Nanostructured cobalt and manganese oxide clusters as efficient water oxidation catalysts. Energy Environ. Sci. 2010, 3, 1018-1027. [CrossRef]

48. Hummers, W.S., Jr.; Offeman, R.E. Preparation of graphitic oxide. J. Am. Chem. Soc. 1958, 80, 1339. [CrossRef]

49. Choudhury, A. Polyaniline/silver nanocomposites: Dielectric properties and ethanol vapour sensitivity. Sens. Actuators B 2009, 138, 318-325. [CrossRef]

50. Azarang, M.; Shuhaimi, A.; Yousefi, R.; Moradi Golsheikh, A.; Sookhakian, M. Synthesis and characterization of $\mathrm{ZnO} \mathrm{NPs} /$ reduced graphene oxide nanocomposite prepared in gelatin medium as highly efficient photo-degradation of MB. Ceram. Int. 2014, 40, 10217-10221. [CrossRef]

51. Pham, V.H.; Cuong, T.V.; Hur, S.H.; Oh, E.; Kim, E.J.; Shin, E.W.; Chung, J.S. Chemical functionalization of graphene sheets by solvothermal reduction of a graphene oxide suspension in N-methyl-2-pyrrolidone. J. Mater. Chem. 2011, 21, 3371-3377. [CrossRef]

52. Gopalakrishnamurthy, H.; Rao, M.S.; Kutty, T.N. Thermal decomposition of titanyl oxalates-I: Barium titanyl oxalate. J. Inorg. Nucl. Chem. 1975, 37, 891-898. [CrossRef]

53. Rahy, A.; Yang, D.J. Synthesis of highly conductive polyaniline nanofibers. Mater. Lett. 2008, 62, 4311-4314. [CrossRef]

54. Shi, L.; Wang, X.; Lu, L.; Yang, X.; Wu, X. Preparation of $\mathrm{TiO}_{2}$ / polyaniline nanocomposite from a lyotropic liquid crystalline solution. Synth. Metals 2009, 159, 2525-2529. [CrossRef]

55. Mahanta, D.; Madras, G.; Radhakrishnan, S.; Patil, S. Adsorption of sulfonated dyes by polyaniline emeraldine salt and its kinetics. J. Phys. Chem. B 2008, 112, 10153-10157. [CrossRef] [PubMed]

(C) 2016 by the authors; licensee MDPI, Basel, Switzerland. This article is an open access article distributed under the terms and conditions of the Creative Commons Attribution (CC-BY) license (http://creativecommons.org/licenses/by/4.0/). 